\title{
N6-isopentenyladenosine dual targeting of AMPK and Rab7 prenylation inhibits melanoma growth through the impairment of autophagic flux
}

\author{
Roberta Ranieri ${ }^{1}$, Elena Ciaglia ${ }^{1}$, Giuseppina Amodio ${ }^{1}$, Paola Picardi ${ }^{2,5}$, Maria Chiara Proto ${ }^{2}$, Patrizia Gazzerro ${ }^{2}$, Chiara Laezza ${ }^{3,4}$, \\ Paolo Remondelli ${ }^{1}$, Maurizio Bifulco ${ }^{1,4,6}$ and Simona Pisanti ${ }^{1,6}$
}

Targeting the autophagic process is considered a promising therapeutic strategy in cancer since a great number of tumors, including melanoma, show high basal levels of protective autophagy that contributes to tumor progression and chemoresistance. Here, exploiting both in vitro and in vivo approaches, we identified N6-isopentenyladenosine (iPA), an end product of the mevalonate pathway, as a novel autophagy inhibitor with an interesting anti-melanoma activity. iPA, after being phosphorylated by adenosine kinase into 5'-iPA-monophosphate, induces autophagosome accumulation through AMPK activation, measured by increased fluorescent GFP-LC3 puncta and enhanced conversion into the lipidated autophagosome-associated LC3-II. However, at a later stage iPA blocks the autophagic flux monitored by p62 accumulation, Luciferase reporter-based assay for LC3 turnover in living cells and fluorescence of a tandem RFP-GFP-LC3 construct. Impaired autophagic flux is due to the block of autophagosome-lysosome fusion through the defective localization and function of Rab7, whose prenylation is inhibited by iPA, resulting in a net inhibition of autophagy completion that finally leads to melanoma apoptotic cell death. AMPK silencing prevents apoptosis upon iPA treatment, whereas basal autophagosome turnover is still inhibited due to unprenylated Rab7. These results strongly support the advantage of targeting autophagy for therapeutic gain in melanoma and provide the preclinical rational to further investigate the antitumor action of IPA, able to coordinately induce autophagosome accumulation and inhibit the autophagic flux, independently targeting AMPK and Rab7 prenylation. This property may be particularly useful for the selective killing of tumors, like melanoma, that frequently develop chemotherapy resistance due to protective autophagy activation.

Cell Death and Differentiation (2018) 25, 353-367; doi:10.1038/cdd.2017.165; published online 13 October 2017

Cutaneous melanoma, although accounting for only the $5 \%$ of all cancer types, is the deadliest form of skin cancer with a very unfavorable prognosis when metastatic and with a median progression free-survival at the stage IV of less than 1 year. ${ }^{1}$ It is characterized by a high mutational rate of key oncogenes, which results in a wide range of molecular alterations responsible for its unresponsiveness to conventional chemotherapy and frequent resistance development. ${ }^{2}$ Despite this, novel chemotherapy and immunotherapy protocols employing inhibitors of key proteins of the MAPK pathway such as BRAF, mutated in the $40-60 \%$ of melanomas, and the immune checkpoints PD- 1 and CTLA-4, have undoubtedly improved the treatment of advanced melanoma. ${ }^{3,4}$ However, a substantial long-term survival benefit has not yet been reached and such novel therapies are also associated with the occurrence of resistance almost in all cases within a year and severe treatment-related adverse reactions that considerably narrow the spectrum of responsive patients. ${ }^{5,6}$ For these reasons and taking into account the growing incidence of melanoma worldwide in the last years, it is imperative to discover new targets and elaborate more effective and lasting therapeutic strategies.
Autophagy can be considered as a quality control mechanism crucial to preserve cellular homeostasis and viability under stressful conditions. ${ }^{7}$ Its dysfunction may result in several human diseases, such as neurodegenerative disorders, cardiomyopathies and cancer. ${ }^{8}$ Autophagy has a pivotal but complex role in cancer, preventing tumorigenesis in the early stages through the protection of cells from genetic instability, metabolic stress and chronic inflammation. Conversely, it promotes cancer cell survival in established or advanced tumors, providing the substrates to sustain tumor metabolism and growth and protect from drug-induced stress. ${ }^{9,10}$ A great number of tumors, including melanoma, show high basal levels of autophagy linked to tumor aggressiveness, weak response to chemotherapy, poor outcome and reduced survival. ${ }^{11}$ Therefore, a deliberate autophagy inhibition strategy, in combination with classic chemotherapy and immunotherapy protocols, could be useful to improve the therapeutic efficacy and contrast chemoresistance.

N6-isopentenyladenosine (iPA) is a product of the mevalonate pathway, constituted by an adenosine moiety modified at the nitrogen in the position 6 of the purine base with an

\footnotetext{
${ }^{1}$ Department of Medicine, Surgery and Dentistry 'Scuola Medica Salernitana', University of Salerno, Salerno, Italy; ${ }^{2}$ Department of Pharmacy, University of Salerno, Salerno, Italy and ${ }^{3}$ Institute of Endocrinology and Experimental Oncology (IEOS), Consiglio Nazionale delle Ricerche (CNR), Naples, Italy

*Corresponding author: M Bifulco or S Pisanti Department of Medicine, Surgery and Dentistry 'Scuola Medica Salernitana', University of Salerno, Via Salvatore Allende, Baronissi (SA) 84081, Italy. E-mail: mbifulco@ unisa.it or Tel: +390 899651 15; E-mail: spisanti@ unisa.it

${ }^{4}$ Current address: Department of Molecular Medicine and Medical Biotechnology (DMMBM), University of Naples Federico II, Via S. Pansini 5, 80131, Naples, Italy.

${ }^{5}$ Current address: Axxam Spa OpenZone, via A. Meucci, 3-20091 Bresso, Milano, Italy

${ }^{6}$ These authors are considered co-last authors.

Received 03.5.17; revised 03.8.17; accepted 06.8.17; Edited by H-U Simon; published online 13.10.17
} 
isopentenyl chain derived from the mevalonate precursor dimethylallyl pyrophosphate, which is in equilibrium with its isomer, isopentenyl pyrophosphate. ${ }^{12-16}$ The exact role of free iPA inside mammalian cells has not been fully disclosed. ${ }^{17}$ The interest in the study of the biological properties of this compound has been strengthened by several experimental observations regarding a promising antitumor efficacy in preclinical models of different malignant tumors exerted through multiple mechanisms of action including the inhibition of proliferation, the block of cell cycle and the induction of apoptosis. ${ }^{18-23}$ Among the possible iPA biochemical targets proposed, the inhibition of farnesyl diphosphate synthase (FDPS) and hence of protein prenylation is the most supported. ${ }^{18,24}$ More recently, we reported that iPA is monophosphorylated into 5'-iPA-monophosphate (iPAMP) by the adenosine kinase (ADK) and in this form it directly activates AMP-activated protein kinase (AMPK) in endothelial cells, thus inhibiting the angiogenic process both in vitro and in vivo. ${ }^{25}$ AMPK, a sensor of energy status that regulates cellular energy homeostasis, is activated by stimuli that augment the AMP/ATP ratio or by AMP mimetics, resulting in the inhibition of protein synthesis and in the activation of autophagy. ${ }^{26}$

Since autophagy is a mechanism that could be successfully targeted for therapeutic gain in melanoma patients and given the antitumor properties of iPA and its ability to target AMPK, in the present study we investigated for the first time the involvement of the autophagic process in the antitumor action of iPA in both in vitro and in vivo models of melanoma.

\section{Results}

iPA inhibits melanoma cell proliferation and colony formation inducing G1-phase stasis. At first, the direct effect of IPA on A375 melanoma cells viability was investigated. iPA inhibited time- and dose-dependently melanoma cell proliferation in the low micromolar range (Figure 1a, black bars). The time-dependency was lost at longer time points. In order to investigate the mechanism through which iPA exerted its inhibitory effects and to confirm the phosphorylation in position $5^{\prime}$ - by ADK as pivotal for IPA action, ${ }^{25}$ melanoma cells were pre-treated with an ADKspecific inhibitor, 5-Itu $(30 \mathrm{nM}, 30 \mathrm{~min})$. The pre-treatment with 5-Itu (Figure 1a, gray bars) prevented the effects of iPA on melanoma cell proliferation at all the concentrations and time points, confirming that the active metabolite is actually N6-isopentenyladenosine 5'-monophosphate (iPAMP) and therefore that the phosphorylation of iPA in C5 by ADK results to be crucial also to the antitumor activity of iPA. Also prolonged exposure to iPA inhibited colony formation in a concentration-related manner, reducing the number and dimension of colonies. Once again, the pre-treatment with 5-Itu completely reverted the inhibitory effect of iPA on longterm colony formation (Figure $1 \mathrm{~b}$ ). To analyze the precise nature of the antiproliferative action of iPA, melanoma cell cycle distribution was evaluated. As shown by quantitative analysis, iPA blocked proliferation of melanoma cells in G1 phase already after $24 \mathrm{~h}$ of treatment at the highest concentration tested and this effect was increased after
$48 \mathrm{~h}$ of treatment (Figure 1c). After $72 \mathrm{~h}$ of treatment the $\mathrm{G} 1$ phase stasis was no more relevant. These data demonstrate that iPA displays an inhibitory activity in the low micromolar concentration range on both short- and long-term melanoma cell growth.

iPA induces autophagosome accumulation through AMPK activation and inhibition of the mTOR pathway. We previously demonstrated that iPA was able to activate the AMPK pathway in endothelial cells, thus inhibiting the angiogenic process. ${ }^{25}$ To investigate whether iPA was able to activate the AMPK pathway also in melanoma cells, phosphorylation at Thr-172 of the AMPK-a catalytic subunit was evaluated as a read-out of its activity. A dose-dependent induction of AMPK phosphorylation was detected at $24 \mathrm{~h}$, whereas the total levels of AMPK protein were not affected (Figure 2a). We next analyzed the effect of iPA on mTOR, a downstream effector of AMPK, showing that iPA caused a dose-dependent inhibition of mTOR phosphorylation, thus inhibiting its function. Further, we examined one of the main mTOR targets, p70S6K, whose phosphorylation at Ser-371 directly reflects mTOR activity. We observed, also in this case, a dose-dependent inhibition of phosphorylation, particularly evident at the $10 \mu \mathrm{M}$ dose (Figure 2a). Since it is wellestablished that the AMPK pathway, also through mTOR downregulation, is a key regulator of cellular responses including autophagy, we next investigated if iPA might affect the autophagic process. A375 cells, as several melanoma cell lines and primary melanoma lesions, are characterized by basal levels of autophagy activation. ${ }^{9}$ During autophagy, the marker LC3 (microtubule-associated protein-1 light chain3) translocates from the cytosol into the autophagosomes, thus redistributing from a diffuse cytoplasmic location to discrete puncta. As shown in Figure $2 \mathrm{~b}$ a redistribution of GFP-LC3 from a diffuse cytoplasmic location to a punctate fluorescence occurred upon iPA treatment. The number of fluorescent puncta and also the number of total cells with punctate GFP-LC3 fluorescence increased in a dosedependent manner in treated cells with respect to the control. LC3 translocation into autophagosomes was further confirmed biochemically through western blot, examining the conversion of the non-lipidated LC3-I into the lipidated autophagosome-associated form LC3-II. We found that IPA enhanced the accumulation of LC3-II in a time- and dosedependent manner (Figure 2c). All together these data suggest that iPA treatment results in autophagosome accumulation in melanoma cells.

Autophagosome accumulation is due to autophagic flux inhibition by iPA. It has been extensively demonstrated that an induction of LC3-II expression and increased numbers of autophagosomes can be due to the activation of autophagy that leads to an increased autophagosome production but also to the inhibition of the autophagic process at a later step, resulting in a reduction of autophagosome turnover. In order to discern among these two different possibilities, the next step was to measure the autophagic flux. At first, we checked through immunoblot the protein levels of p62/SQSTM1, a sensitive autophagy substrate implicated in autophagic cargo recognition, that is degraded in the later stages of autophagy, 

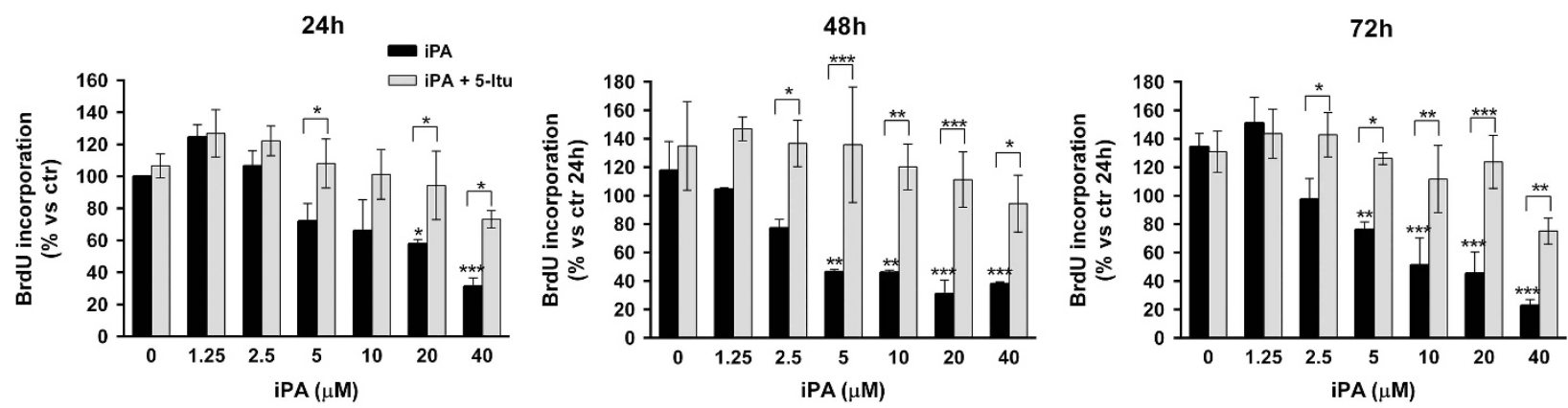

b

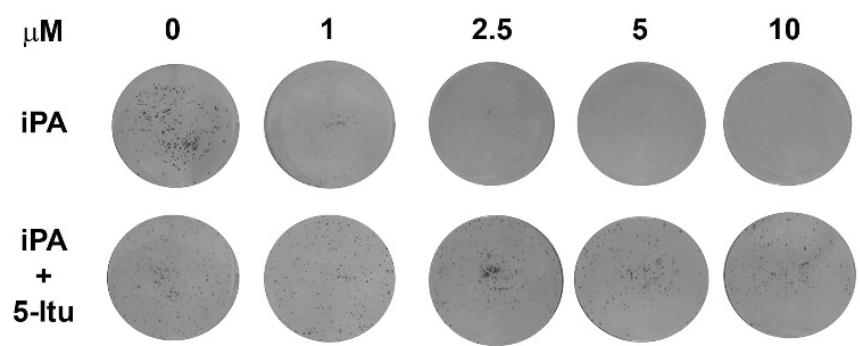

C
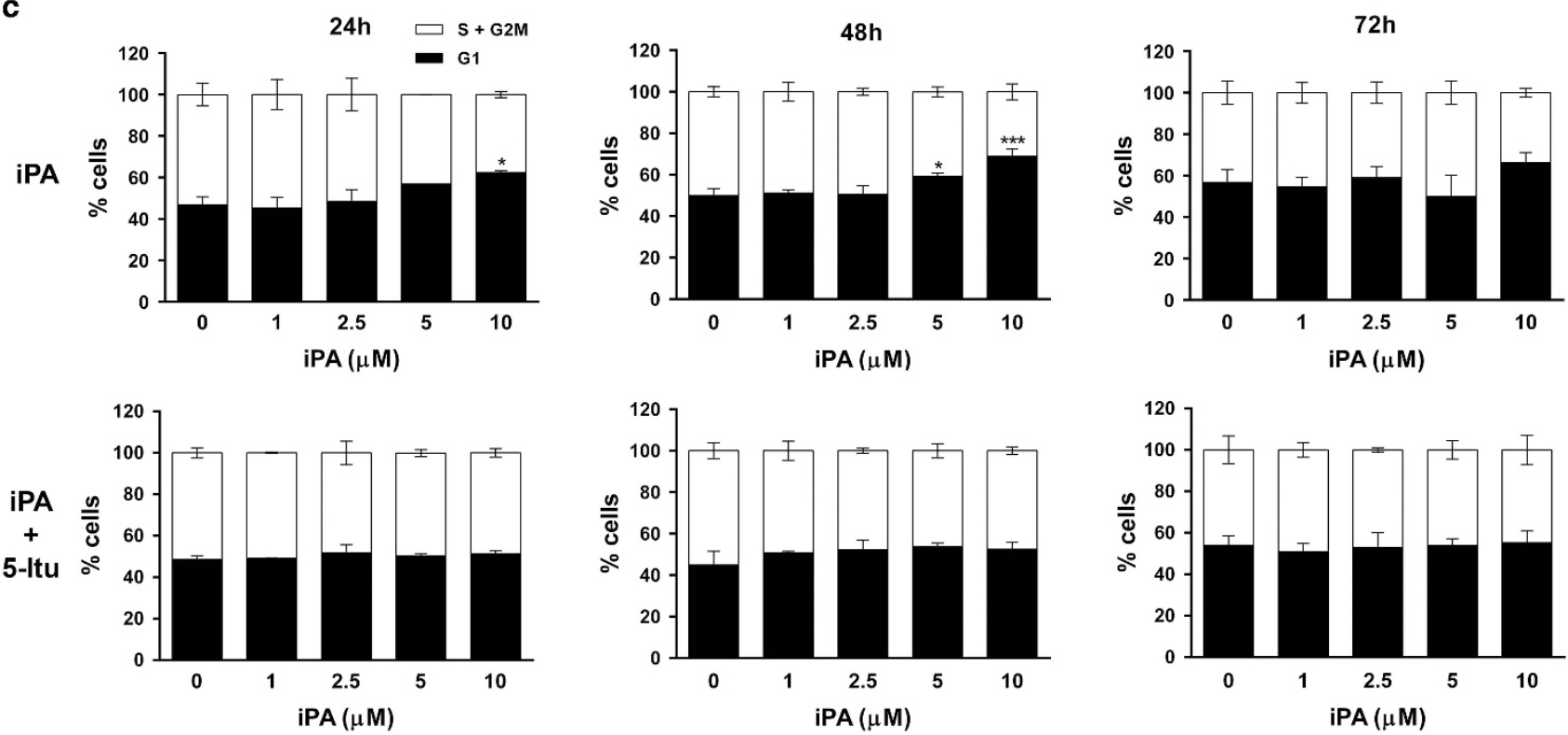

Figure 1 iPA inhibits melanoma cell proliferation and colony formation inducing a G1-phase stasis. Melanoma cells were treated with increasing concentrations of iPA alone (from 1 to $40 \mu \mathrm{M}$ ) or following 5-Itu pre-treatment ( $30 \mathrm{nM}, 30 \mathrm{~min}$ ) for the indicated time points. (a) Histograms show the inhibition of proliferation of melanoma cells, pre-treated (shaded bars) or not (black bars) with 5-Itu, performed by BrdU proliferation assay for 24, 48 and $72 \mathrm{~h}$. (b) The image shows representative pictures of melanoma cells after 10 days of treatment with increasing concentrations of IPA (1-10 $\mu \mathrm{M})$ pre-treated or not with 5 -Itu ( $30 \mathrm{nM}, 30 \mathrm{~min})$. Colonies have been fixed, stained with crystal violet and photographed. The experiment was performed three times in triplicate. (c) The histograms report the quantitative analysis of melanoma cells in the different phases of cell cycle following treatment with iPA (from 1 to $10 \mu \mathrm{M}$ ) with or without pre-treatment with 5 -ltu ( $30 \mathrm{nM}, 30 \mathrm{~min}$ ) in time course (24, 48 and $72 \mathrm{~h}$ ), expressed as percentage versus control. (a and c) Results are expressed as mean \pm S.D. of three experiments performed in triplicate (ANOVA, ${ }^{\star} P<0.05,{ }^{* \star} P<0.01,{ }^{* \star} P<0.001$ versus control, represented by cells untreated or treated without 5 -ltu)

reflecting autophagosome turnover. Consistent with this notion, we observed a time- and dose-dependent accumulation of p62 upon iPA treatment, that is suggestive of autophagic flux inhibition (Figure 2c). This phenomenon was further confirmed by confocal microscopy analysis. iPA treatment led to a dose-dependent accumulation of p62 and GFP-LC3 puncta in big spots, mainly concentrated near the nucleus, that showed extensive co-localization, suggesting that iPA inhibited autophagic flux at a late stage (Figure 3a).

In order to clarify the reason of iPA-induced accumulation of LC3-II and GFP-LC3 positive puncta, we measured autophagic flow by employing a Renilla luciferase (RLuc) reporterbased assay for LC3 turnover in living cells. ${ }^{27}$ This assay allows to compare the rate of LC3 degradation during the 
a

$$
\begin{aligned}
& \text { Time } 24 \mathrm{~h} \quad \mathrm{kDa} \\
& \text { p-AMPK }=62 \\
& \text { AMPK }-\cdots-- \\
& \text { Tubulin }--\cdots \\
& 012.5510 \\
& \text { iPA }(\mu \mathrm{M})
\end{aligned}
$$
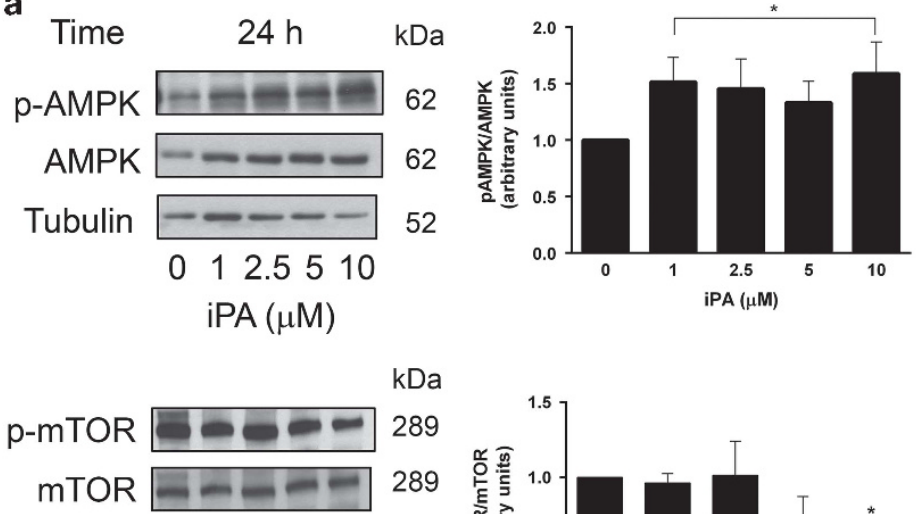

$\mathrm{kDa}$

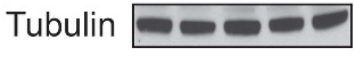
289 289

p-p70 $-0---$

Tubulin

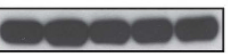

iPA $(\mu \mathrm{M}) \quad 012.5510$
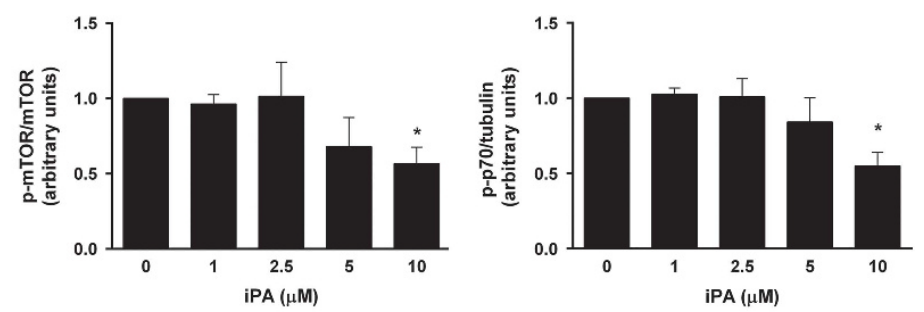

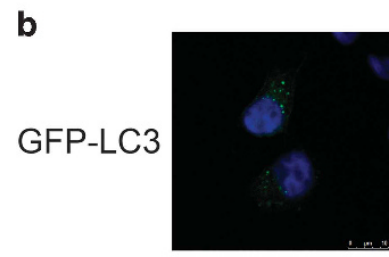

CTR

c

iPA $(\mu \mathrm{M}) \quad 0 \quad 12.5510$

c

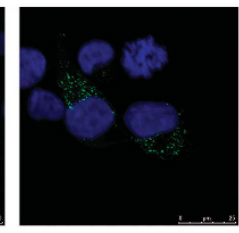

1

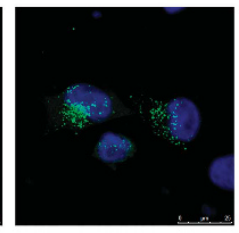

2.5

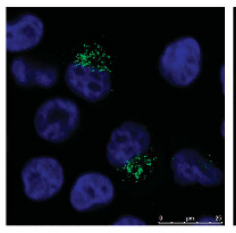

5

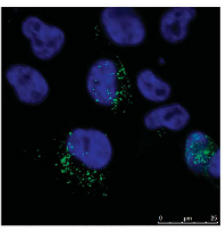

$\mathrm{HCQ}$

iPA $(\mu \mathrm{M})$

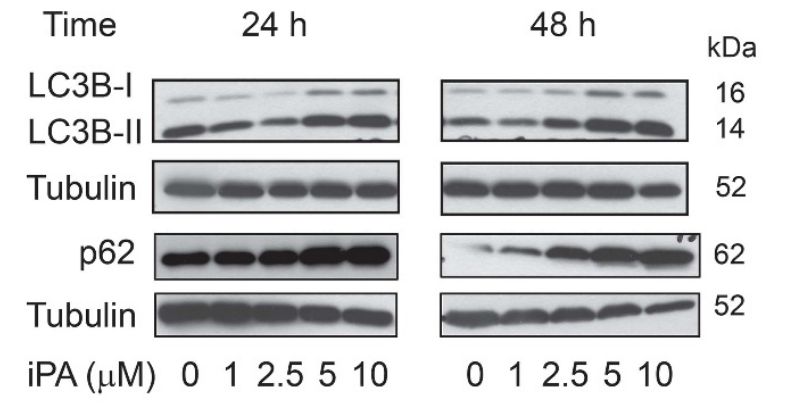

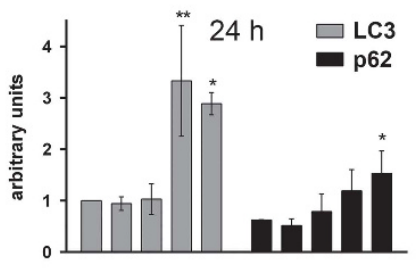

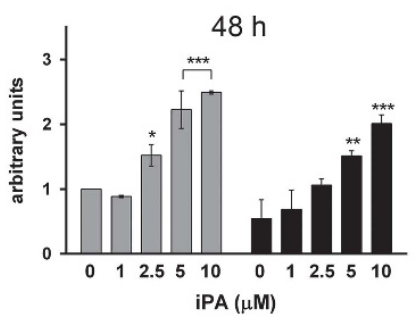

Figure 2 iPA induces AMPK activation and inhibition of the mTOR pathway resulting in autophagosome accumulation. (a) Representative western blots showing the expression of p-AMPK (Thr-172), AMPK, p-mTOR (Ser-2448), mTOR, p-p70 (Ser-371) of melanoma cells treated with iPA at the indicated concentrations for $24 \mathrm{~h}$. Tubulin served as the loading control. Data are representative of three different experiments with similar results. Histograms (right panels) report the results of the quantitative analysis (arbitrary units) as mean \pm S.D. of three independent experiments (ANOVA, ${ }^{*} P<0.05$ versus control). (b) To visualize autophagy, melanoma cells were transiently transfected with green fluorescent protein (GFP)-tagged LC3, a marker of the autophagic process. Representative images of A375 cells expressing GFP-LC3 (green) upon iPA treatment at different concentrations $(1,2.5,5 \mu \mathrm{M})$. Hydroxychloroquine (HCQ) treatment $(10 \mu \mathrm{M})$ was used as positive control. Nuclei were counterstained with DAPI. Representative pictures are shown. Original magnification: x63. Scale bars: $25 \mu \mathrm{M}$. (c) Melanoma cells were treated with iPA at the indicated concentrations for 24 and $48 \mathrm{~h}$. The autophagic markers LC3B and p62 were analyzed by western blot. Tubulin served as a loading control. The data shown are representative of three experiments with similar results. The histograms (right panels) report the quantitative analysis (arbitrary units) expressed as mean \pm S.D. of three independent experiments (ANOVA, ${ }^{*} P<0.05,{ }^{* *} P<0.01,{ }^{* * *} P<0.001$ versus control) 

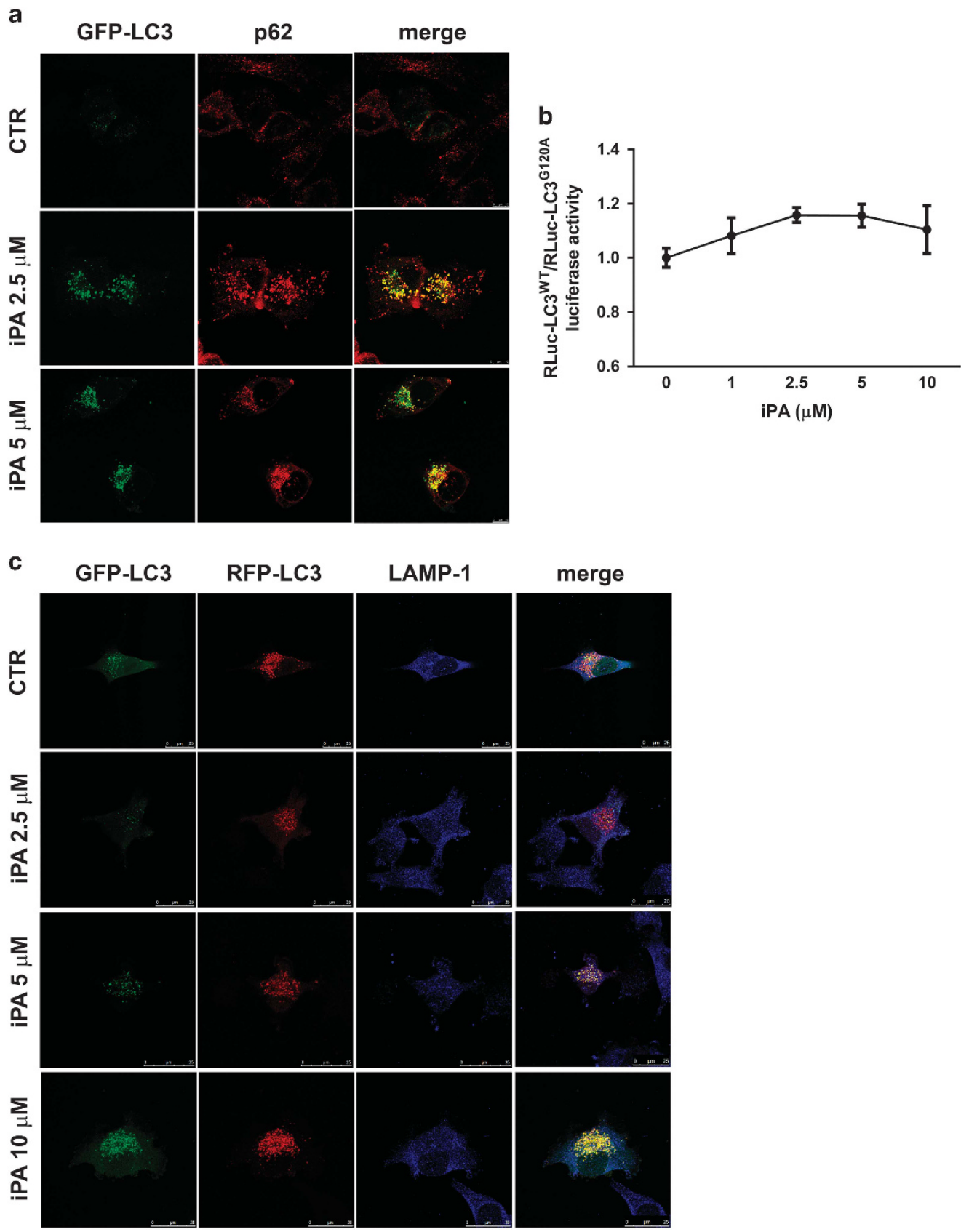

Figure 3 iPA inhibits autophagic flux. (a) A375 cells transiently expressing GFP-LC3 were treated with increasing concentrations of iPA $(2.5,5 \mu \mathrm{M})$ for $48 \mathrm{~h}$, followed by staining with anti-p62 (red). Representative pictures are shown. Original magnification: $\times 63$. Scale bars: $25 \mu \mathrm{M}$. (b) RLuc-LC3 ${ }^{\mathrm{WT}}$ and RLuc-LC3 ${ }^{\mathrm{G} 120 \mathrm{~A}}$ transfected cells were cultured for $48 \mathrm{~h}$ and then treated in parallel with increased concentrations of iPA. Luciferase activity was detected as described in Materials and Methods using the luciferase substrate coelenterazine (EnduRen), optimized for use in living cells, allowing a live cell kinetic analysis of autophagic flux in our assay system. Data represent the mean \pm S.D. of three independent experiments performed in triplicates. (c) A375 cells transiently expressing RFP-GFP-LC3 construct were treated with increasing concentrations of iPA (2.5, 5 , $10 \mu \mathrm{M})$ for $48 \mathrm{~h}$, followed by staining with anti-LAMP-1 (blue). Representative pictures are shown. Original magnification: $\times 63$. Scale bars: $25 \mu \mathrm{M}$

autophagic process in melanoma cells expressing RLuc fused to either wild-type LC3 or mutant LC3 (G120A) that is unable to undergo to cleavage and lipidation-dependent changes. Autophagic flux was not induced by iPA since there was not a lipidation-dependent gradual loss of RLuc-LC3, as observed also with $\mathrm{HCQ}$ treatment, a known inhibitor of autophagy $\left(\right.$ RLuc-LC3 $\left.{ }^{\mathrm{WT}} /{ }^{\mathrm{G} 120}=1.12\right)$ (Figure $\left.3 b\right)$.
Another powerful way to monitor the autophagic flux, evaluating at the same time the fusion of autophagosomes with lysosomes, employs the measurement of the fluorescence change of a tandem RFP-GFP-LC3 construct, which allows to distinguish autophagosomes from autolysosomes. Indeed, upon the fusion of autophagic vesicles with lysosomes the GFP fluorescence is quenched by the acidic environment, 
Figure 4 iPA impairs autophagic flux through the inhibition of autophagosome-lysosome fusion. (a) Flow cytometric analysis of LysoTracker Red-stained cells after treatment with iPA $(2.5,5,10 \mu \mathrm{M})$ for 24,48 or $72 \mathrm{~h}$. The most representative images of flow cytometric analysis are shown in the left panel, while on the right the histogram reports the quantitative analysis of mean fluorescence intensity (MFI) of LysoTracker Red from three independent experiments at the different time points. (b) Flow cytometric analysis of acridine orange-stained cells upon IPA treatment $(2.5,5,10 \mu \mathrm{M})$ for 24,48 or $72 \mathrm{~h}$. FL1 indicates green color fluorescence (cytoplasm and nucleus) whereas FL3 red color fluorescence (acidic vesicular organelles). In the left panel the most representative images of flow cytometric analysis are shown. The histogram on the right reports the quantitative analysis of FL3-positive cells from three independent experiments at the different time points. (c) The histogram reports the activity of cathepsin B expressed as foldincrease in relative fluorescence units (RFU) compared with the level of negative control sample in cells treated with iPA (from 1 to $10 \mu \mathrm{M}$ ) for $48 \mathrm{~h}$. (d) A375 cells transiently expressing GFP-LC3 were treated with increasing concentrations of IPA $(2.5,5,10 \mu \mathrm{M})$ for $48 \mathrm{~h}$, followed by staining with LysoTracker (blue) and anti-Rab7 (red). HCQ treatment $(10 \mu \mathrm{M})$ was used as control of autophagy inhibition. Representative pictures are shown. Original magnification: $\times 63$. Scale bars: $25 \mu \mathrm{M}$. (e) Melanoma cells were treated with iPA at the indicated concentrations for $48 \mathrm{~h}$. Protein prenylation was analyzed by a change in the electrophoretic mobility of HDJ-2 and Rab7 ( $\mathrm{U}=$ unprenylated; $\mathrm{P}=$ prenylated). Prenylation was also analyzed through the U-Rap1A antibody that specifically detects only the unprenylated form of Rap1A. Total Rap1A levels are reported below. Tubulin served as the loading control. The immunoblots showed are representative of three independent experiments with similar results. Histograms (lower panel) report the results of the quantitative analysis (arbitrary units) as mean \pm S.D. of three independent experiments. (ANOVA, ${ }^{\star} P<0.05$ versus control). (a-c) Results are expressed as mean \pm S.D. of three experiments (ANOVA, ${ }^{*} P<0.05,{ }^{*} P<0.01,{ }^{* \star} P<0.001$ versus control)

whereas the RFP signal is not affected. ${ }^{28}$ iPA treatment led to a dose-dependent increase also of red fluorescence puncta, indicating once again autophagosomes accumulation. However, the treatment resulted in the increasing of yellow puncta (co-localization of GFP and RFP fluorescence in the absence of GFP quenching), and decreasing of red-only puncta and purple puncta (co-localization of RFP-LC3 and LAMP-1, lysosome marker), suggesting an impairment of autophagosome-lysosome fusion (Figure 3c).

All together these data indicate that the accumulation of autophagosomes induced by IPA is due to an impairment of the autophagic flux due to distal autophagy blockade.

\section{iPA impairs autophagic flux inhibiting autophagosome-} lysosome fusion through unprenylated Rab7. A possible reason for the impaired autophagic flux induced by iPA could be the inhibition of fusion of autophagosomes with late endosomes or lysosomes. Since autophagosome-lysosome fusion has been reported to be dependent on the acidic environment, lysosome functionality was evaluated. ${ }^{29}$ To this end, two specific $\mathrm{pH}$ probes, LysoTracker Red and acridine orange, were employed. iPA induced a dose- and timedependent accumulation of acidic vesicles as testified by an increased fluorescence intensity of the two probes (Figures $4 \mathrm{a}$ and b). Lysosomal functionality, monitored by measuring cathepsin B activity through an enzymatic assay, was not deteriorated by iPA treatment that, on the contrary, induced cathepsin B activity in a dose-dependent manner (Figure 4c). Therefore, the inhibition of autophagosomelysosome fusion is not linked to an impaired lysosomal acidification or degradative function. Next, the effects on autophagosome maturation were assessed by measuring the co-localization efficiency of GFP-LC3 labeled autophagosomes with LysoTracker Red-stained lysosomes. As detected, the fusion efficiency of GFP-LC3 with LysoTracker Red lysosomes was inhibited by iPA, differently from $\mathrm{HCQ}$ that is known to inhibit autophagy by raising lysosomal $\mathrm{pH}$ (Figure 4d), confirming the observed inhibition of RFP-LC3 and LAMP-1 co-localization (Figure 3c). The process of how autophagosomes are guided to fuse with lysosomes is one of the crucial steps in autophagy. The completion of autophagosome maturation and the fusion with lysosomes requires the small GTPase Rab7, a prenylated protein that in the active form (Rab-GTP) associates to the membrane. ${ }^{30}$ We already disclosed that IPA inhibits FDPS activity and consequently it affects protein prenylation. ${ }^{18,24}$ iPA treatment inhibited protein prenylation inducing accumulation of unprenylated HDJ-2 and Rap1A (U-Rap1A), as a measure of protein prenylation inhibition (Figure 4e). The effect was specific on prenylation, since the total levels of Rap1A were not affected. More interestingly, as regard to Rab7 prenylation we observed a dose-dependent accumulation of unprenylated Rab7 (U) with respect to the prenylated form $(P)$ upon iPA treatment, detected by a change in electrophoretic mobility by EMSA (Figure 4e). To further understand how iPA blocks the autophagic flux, the intracellular distribution of Rab7 was evaluated. Both GFP-LC3 and Rab7 showed punctate staining in the cytoplasm. Co-localization of both GFP-LC3 and Rab7 and GFP-LC3, Rab7 and Lysotracker, was inhibited by iPA. Upon iPA treatment, unprenylated Rab7 was unable to associate with the membrane and maintained a cytoplasmic punctate localization. Cells treated with $\mathrm{HCQ}$, which does not affect prenylation, showed a different pattern of Rab7 localization mainly concentrated to the membrane in bigger spots and surrounding GFP-LC3 larger aggregates accumulating near to the perinuclear region (Figure 4d). All together our data argue for the inhibition of autophagosomelysosome fusion by iPA through unprenylated Rab7.

Inhibition of autophagy leads to apoptotic cell death. To further examine whether iPA-mediated autophagic flux inhibition eventually affected melanoma cell survival, apoptotic cell death was analyzed. iPA exerted a dose- and timedependent increase in the percentage of apoptotic cells, whereas there were no signs of necrotic cell death (Figure 5a). In order to confirm that phosphorylation into IPAMP by ADK was crucial for IPA pro-apoptotic action and also to indirectly disclose the involvement of AMPK activation in such effects, melanoma cells were pre-treated with 5-Itu that prevented the pro-apoptotic action of iPA (Figure $5 b$ ). The activation of the intrinsic apoptosis pathway was monitored by the analysis of the caspase cascade. Caspase- 9 activation, started from $24 \mathrm{~h}$ upon iPA treatment, followed by caspase-3 activation at $48 \mathrm{~h}$ and resulted in DNA repair enzyme poly(ADP-ribose) polymerase (PARP) cleavage, that finally leads to apoptotic cell death. The activation of such pro-apoptotic proteins by IPA was prevented by 5-Itu (Figure $5 \mathrm{c}$ ). Following 5-Itu pre-treatment iPA was not more 
a

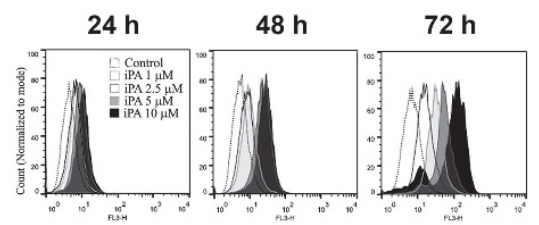

b

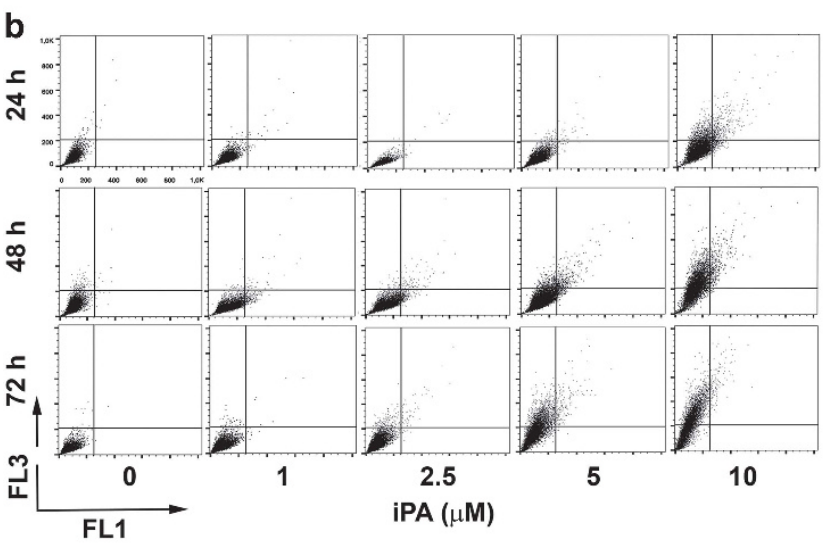

C

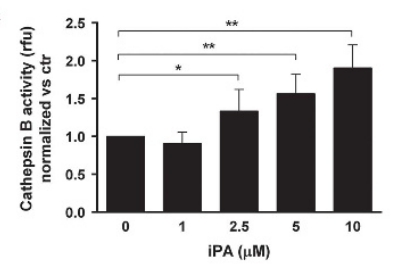

d

GFP-LC3

$\stackrel{\underline{n}}{\mathrm{U}}$

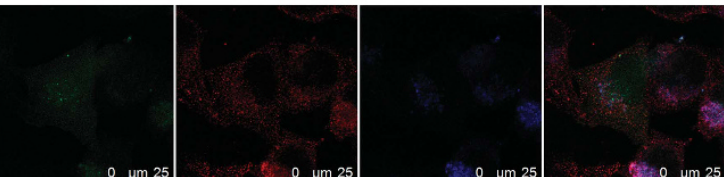

$\sum_{\substack{n \\ \text { Ln }}}$

กับ

ㄴ.

$\sum$

尔

을

ఏ

옴

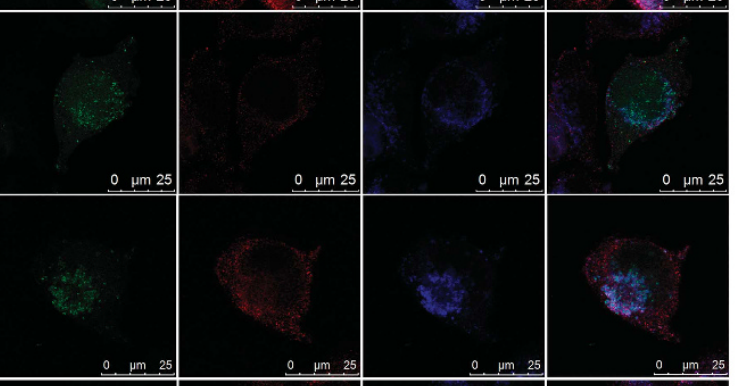

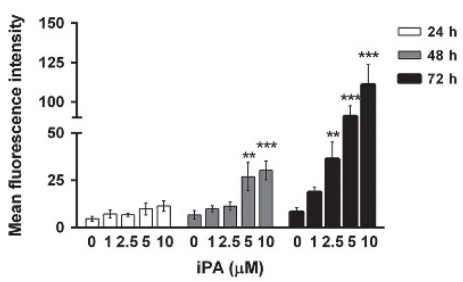

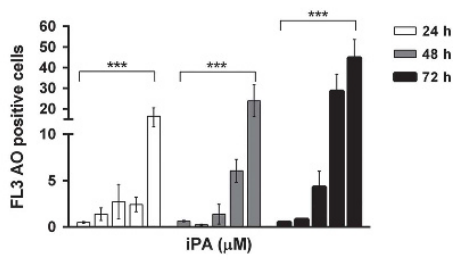

e

HDJ-2 二ニニニー-U 47

Tubulin 52

U-Rap1A $\square 22$

Rap1A

Tubulin 52

Rab7 E=-二-U 23

Tubulin 5

012.5510

iPA $(\mu \mathrm{M})$
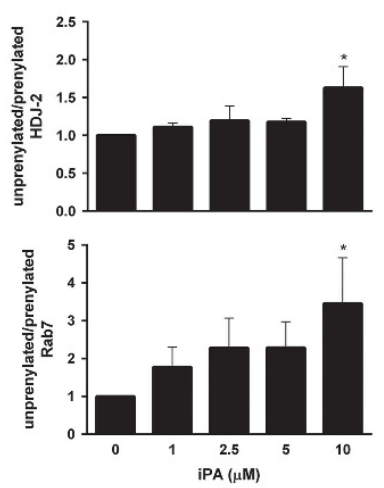
Figure 5 Inhibition of autophagy leads to apoptotic cell death. (a and $\mathbf{b})$ Apoptosis was analyzed by flow cytometry staining with Annexin V and propidium iodide (PI). The histograms report the quantitative flow cytometric analyses of apoptotic melanoma cells (\%) positive for Annexin V (early apoptosis) and Annexin V/PI (late apoptosis) following treatment with IPA (from 1 to $10 \mu \mathrm{M}$ ) in time course (24, 48 and $72 \mathrm{~h}$ ) with (b) or without (a) pre-treatment with 5 -ltu ( $30 \mathrm{nM}, 30$ min). Results are representative of three independent experiments in duplicates. Results are expressed as mean \pm S.D. (ANOVA, ${ }^{*} P<0.05,{ }^{* * *} P<0.001$ versus control). (c) Melanoma cells were treated with iPA at the indicated concentrations for 24 (CASP9) or $48 \mathrm{~h}$ (PARP and CASP3) with or without pre-treatment with 5-Itu ( $30 \mathrm{nM}, 30 \mathrm{~min})$. Protein levels of cleaved CASP9, PARP and CASP3 along with their total protein levels were detected by western blot. Tubulin served as a loading control. When necessary, blots were stripped and reprobed with the different antibodies. Data are representative of three different experiments with similar results. The histograms (right panels) report the quantitative analysis (arbitrary units) expressed as means \pm S.D. of at least three independent experiments with similar results. (d) Melanoma cells were treated with iPA at the indicated concentrations for $24 \mathrm{~h}$ with or without 5 -ltu pre-treatment ( $30 \mathrm{nM}, 30 \mathrm{~min}$ ). Protein levels of p-AMPK (Thr-172), AMPK, LC3B and p62 were analyzed by western blot. Tubulin served as a loading control. When necessary, blots were stripped and reprobed with the different antibodies and for these blots the same tubulin was shown. Data are representative of three different experiments with similar results. The histogram (lower panel) reports the quantitative analysis (arbitrary units) for 5-ltu pre-treated samples, expressed as means \pm S.D. of at least three independent experiments with similar results. The histograms reporting the quantitative analysis of control and iPA curve treatment are already shown in Figure 2a (AMPK) and 2c (LC3, p62). (e) A375 cells transiently expressing GFP-LC3 were pre-treated or not with 5 -Itu ( $30 \mathrm{nM}, 30 \mathrm{~min}$ ) and then treated with iPA (5 $\mu$ M) for $48 \mathrm{~h}$. Samples were then stained with antiLAMP-1 (blue). Representative pictures are shown. Original magnification: $\times 63$. Scale bars: $25 \mu \mathrm{M}$

able to induce phosphorylation and hence activation of AMPK, and the increment of the lipidated autophagosomeassociated form LC3-II and of p62 was lost, confirming iPAMP as the active form of iPA, responsible for the activation of AMPK and subsequently of the autophagic process (Figure 5d). Moreover, in melanoma cells transiently transfected with GFP-LC3 5-Itu impeded the accumulation of LC3 fluorescent puncta induced by iPA. These results indicate that iPA action on both apoptosis and autophagy processes depends on its phosphorylation into the active form iPAMP, suggesting a key role for the AMPK pathway.

\section{iPA effects on autophagosome accumulation and apop- tosis induction are dependent on AMPK activation. On} the basis of the previous findings, to address whether AMPK activation plays an essential role in iPA inhibition of the autophagic flux and subsequent activation of apoptotic cell death, we determined its effects on both autophagy and apoptosis following AMPK a1 catalytic subunit knockdown by small interfering RNA (siRNA) (Figure 6) or pharmacological inhibition by Compound C (Supplementary Figure 1a). AMPK silencing was confirmed by immunoblot of total AMPK expression, which showed at least $50 \%$ of inhibition (Figure 6b). Genetic inhibition of AMPK prevented iPAinduced apoptosis (Figure 6a) evidenced also by cathepsin $B$ activation, implicated in apoptosis (Figure 6b) and by measurement of caspase-3 and PARP cleavage. Further, AMPK siRNA counteracted iPA-induced accumulation of lipidated LC3-II and GFP-LC3 fluorescent puncta that therefore is AMPK-dependent (Figures $6 c$ and $d$ ). The autophagic flux completion, evaluated by $p 62$ levels that remained elevated with AMPK siRNA, was still inhibited by iPA treatment and so independent from AMPK (Figure 6c). As a reason of this, Rab7 distribution, following AMPK silencing and upon iPA treatment, maintained a diffuse punctate cytoplasmic localization typical of the unprenylated inactive form (Figure 6d). Therefore, AMPK signaling pathway is not linked to the inhibition of Rab7 prenylation by iPA. These observations support the evidence that activation of AMPK by iPA is necessary for stimulation of both autophagosomes accumulation and apoptosis but not for the inhibition of Rab7 prenylation and hence for the block of fusion of autophagosomes with lysosomes and the completion of the autophagic process.
iPA inhibits autophagic flux exerting antitumor efficacy in vivo. Finally, in order to confirm a potential antitumor effect of iPA in vivo, melanoma xenografts were generated by subcutaneous inoculation of A375 cells in athymic mice. A rapid and dramatic tumor growth was observed throughout the course of the experiment in the control group, whereas tumors from iPA-treated mice were consistently and significantly smaller (Figure 7a). As anticipated, treatment with iPA significantly enhanced AMPK phosphorylation and activation, reducing $\mathrm{mTOR}$ phosphorylation, as already observed in vitro (Figure 7b). A trend of p62 accumulation was also observed, even if statistical significance was not reached due to high basal p62 levels in one of the control mice (\#4). Moreover, we observed an accumulation of unprenylated Rab7 (U) with respect to the prenylated form $(P)$ (Figure $7 b)$. Overall, these data clearly confirm in vivo the interesting anti-melanoma activity of iPA that acts through AMPK pathway stimulation and inhibition of Rab7 prenylation, resulting in the block of autophagic flux.

\section{Discussion}

The discovery of new effective compounds that may be used with success in melanoma treatment is a fundamental challenge, in particular to treat those tumors that are resistant or non-responsive to the available therapies. To this aim, exploiting both in vitro and in vivo approaches, in this study we demonstrated for the first time the interesting anti-melanoma activity of the isoprenoid derivative iPA. Prior studies have already reported the antitumor action of iPA in different tumor types. ${ }^{18-23,31}$ In addition to a direct antitumor activity, iPA displays pleiotropic properties able to interfere with tumor growth at different levels, being also an angiogenesis inhibitor and an immunomodulator that selectively activates natural killer cells. ${ }^{25,32,33}$ However, the effect of iPA on the autophagic process has never been previously recognized. In the present study we have identified and characterized iPA as a novel autophagy inhibitor that causes a substantial accumulation of autophagosomes followed by inhibition of the autophagic flux and final induction of melanoma cell death both in vitro and in vivo. Targeting the autophagic process is currently considered a promising therapeutic strategy in cancer and its monitoring has been also proposed to have a prognostic relevance. ${ }^{34}$ Indeed, while autophagy suppresses tumorigenesis at early stages, in a great numbers of advanced tumors, 
a

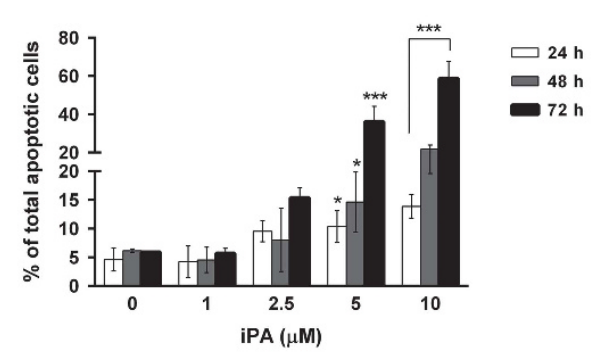

C

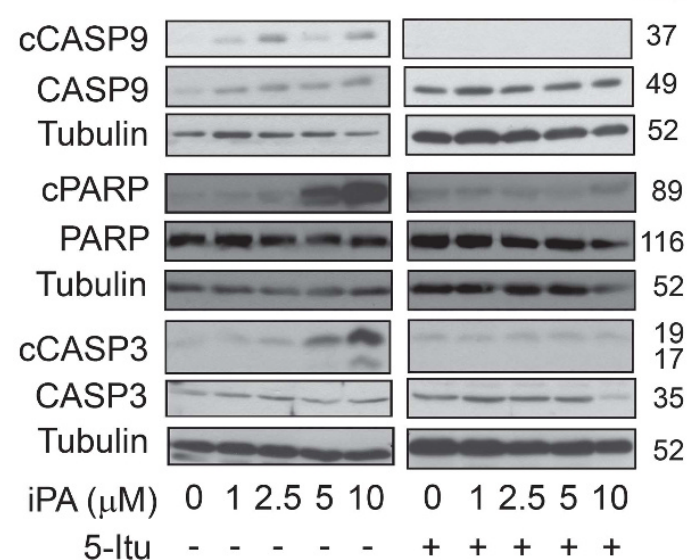

d
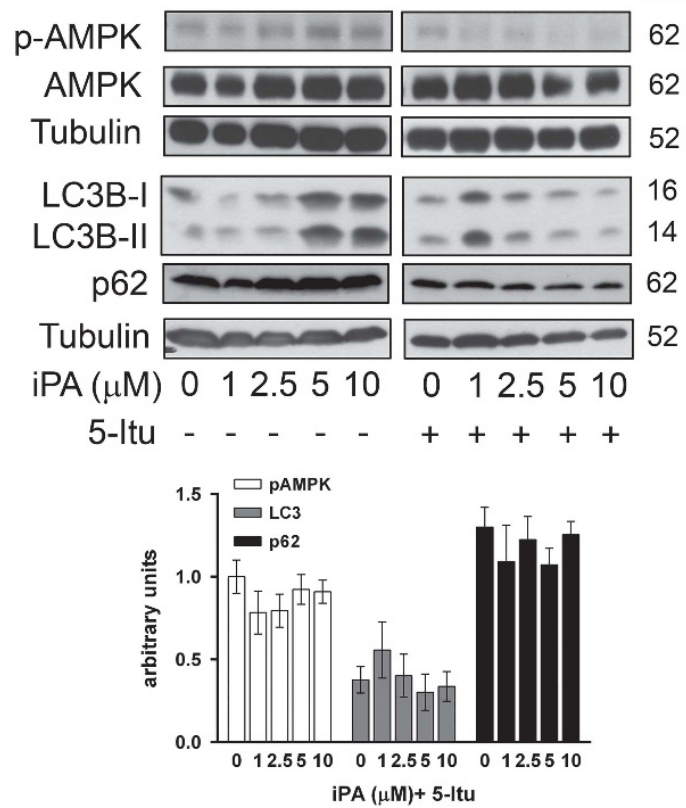

including melanoma, it promotes progression being also responsible of chemo- and radio-resistance. ${ }^{35,36} \mathrm{~A}$ high autophagic index in melanoma correlates to a worse prognosis, with higher invasiveness, lympho nodes metastases, poor response to chemotherapy and reduced survival. ${ }^{11,37}$ For these reasons, autophagy inhibition could
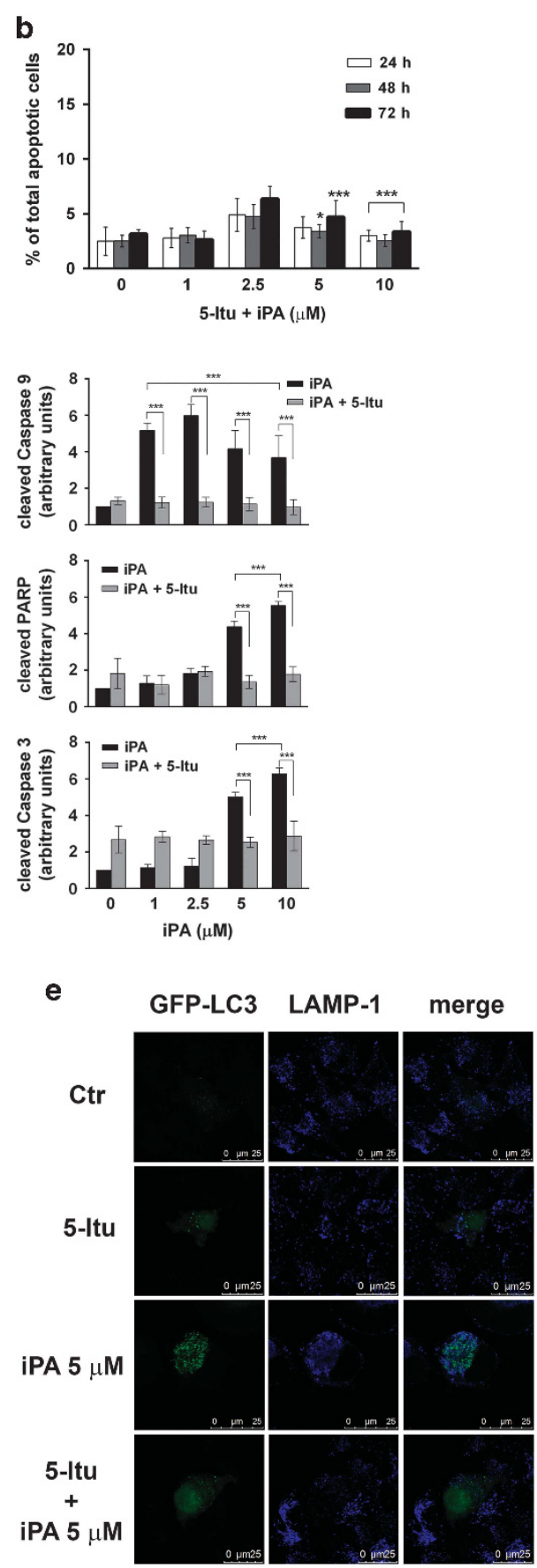

be a successful strategy for therapeutic gain in melanoma patients. Most well-established autophagy inhibitors evaluated in the clinic in combination with chemotherapeutic drugs are $\mathrm{HCQ}$ derivatives that act increasing lysosome basicity causing its dysfunction. ${ }^{38,39}$ Recent screening of natural compounds led to the discovery of elaiophylin, a macrolide antibiotic with a 
a

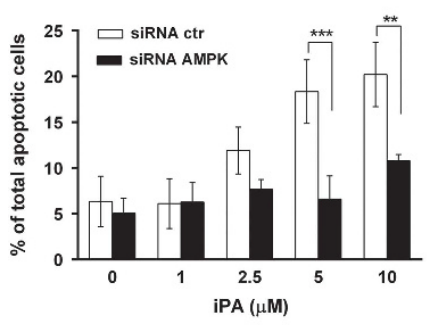

d

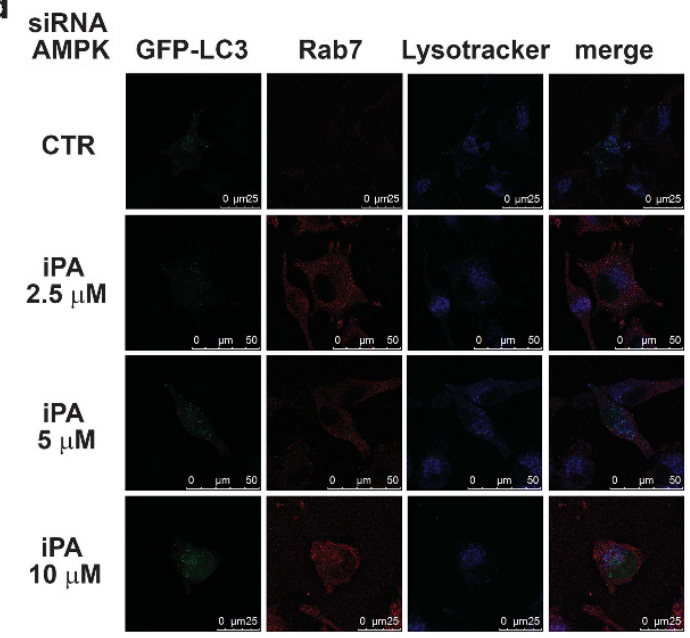

C

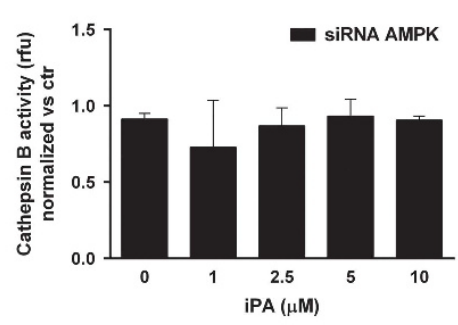

$\mathrm{kDa}$

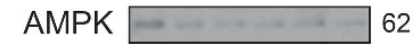

LC3B-I —- 16

LC3B-II $-\ldots-\cdots 14$

p62 $----\longrightarrow 62$

Tubulin 52

iPA $(\mu \mathrm{M}) \quad 0 \quad 0 \quad 12.5510$

SiRNAAMPK -+++++

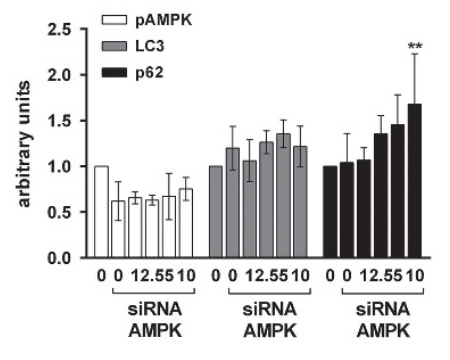

Figure 6 iPA effects on autophagosome accumulation and apoptosis induction are dependent on AMPK activation. (a) The histogram reports the quantitative flow cytometric analysis of apoptotic melanoma cells (\%) positive for Annexin V and Annexin V/PI following treatment with control siRNA or selective AMPK siRNA for $48 \mathrm{~h}$ and subsequently treated with iPA (from 1 to $10 \mu \mathrm{M}$ ) for additional $48 \mathrm{~h}$. Results are representative of three independent experiments in duplicates and are expressed as mean \pm S.D. (ANOVA, ${ }^{* * *} P<0.001$ versus control). (b) The histogram reports the activity of cathepsin B expressed as fold-increase in relative fluorescence units (RFU) compared with the level of negative control sample in cells silenced for AMPK expression by siRNA treatment for $48 \mathrm{~h}$, upon treatment with iPA (from 1 to $10 \mu \mathrm{M}$ ) for additional $48 \mathrm{~h}$. Results are representative of three independent experiments in triplicates and are expressed as mean \pm S.D. (ANOVA). (c) Melanoma cells exposed to control siRNA or selective AMPK siRNA for $48 \mathrm{~h}$ were subsequently treated with iPA at the indicated concentrations for additional $48 \mathrm{~h}$. Protein levels of AMPK, LC3B and p62 were analyzed by western blot. Tubulin served as a loading control. When necessary, blots were stripped and reprobed with the different antibodies. Data are representative of three different experiments with similar results. The histogram (lower panel) reports the quantitative analysis (arbitrary units) expressed as means \pm S.D. of at least three independent experiments with similar results (ANOVA, ${ }^{* *} P<0.001$ ). (d) A375 cells transiently expressing GFP-LC3 were silenced by siRNA for AMPK for $24 \mathrm{~h}$ and then were treated with iPA (from 2.5 to $10 \mu \mathrm{M}$ ) for additional $48 \mathrm{~h}$. Samples were stained with LysoTracker (blue) and anti-Rab7 (red). Representative pictures are shown. Original magnification: $\times 63$. Scale bars: 25 or $50 \mu \mathrm{M}$ (as reported in each picture)

chemical structure distinct from $\mathrm{HCQ}$, able to block autophagic flux by impairment of lysosomal cathepsin activity and exert antitumor activity in ovarian cancer. ${ }^{40}$ Here, we reported that also iPA is a late-stage inhibitor of autophagy, preventing the autophagosome-lysosome fusion through unprenylated Rab7, thus blocking the autophagic flux without affecting lysosome acidity and functionality. A proper autophagic flux is indeed determined by the equilibrium between autophagosome formation and clearance by lysosomes. Rab7 is a small GTP-binding protein that peripherally associates with membranes through a geranylgeranyl lipid tail derived from the isoprenoid pathway. Its GTP-bound form is generally thought to mediate its functions through specific interactions with effector proteins, whereas the inactive form Rab-GDP resides in the cytosol. ${ }^{30}$ Active Rab7 is required for the maturation of late autophagosomes and fusion with lysosomes. Overexpression of a dominant-negative Rab7 mutant in mammalian cells leads to the accumulation of large autophagosomes, caused by autophagic flux interruption. ${ }^{41}$ Moreover, inhibition of Rab geranylgeranyl transferase (GGTase II) and hence of
Rab prenylation results in a net block of autophagy. ${ }^{42}$ Consistent with these observations, our study revealed that there is a significant increase not only in the number but also in the size of autophagosomes in cells treated with iPA, suggesting that autophagosome accumulation is caused by iPA-induced defects in the late autophagosome-lysosome fusion step of autophagy due to a defective localization and function of Rab7 caused by its defective prenylation. Indeed, we already reported that iPA inhibits the activity of FDPS and consequently it may affect prenylation of target proteins like small GTPases. ${ }^{18,24}$ We confirmed that iPA treatment dosedependently inhibits protein prenylation preventing a proper localization and function of Rab7 that shows a punctate cytoplasmic distribution and does not colocalize with both autophagosome and lysosome markers (LC3 and LysoTracker). It is possible however that other members of the Rab family, which may function in parallel or synergistically with Rab7 in autophagosome maturation, may be also involved in iPA action. Indeed, both statins and Rab11 knockdown have been reported to inhibit autophagic flux at 


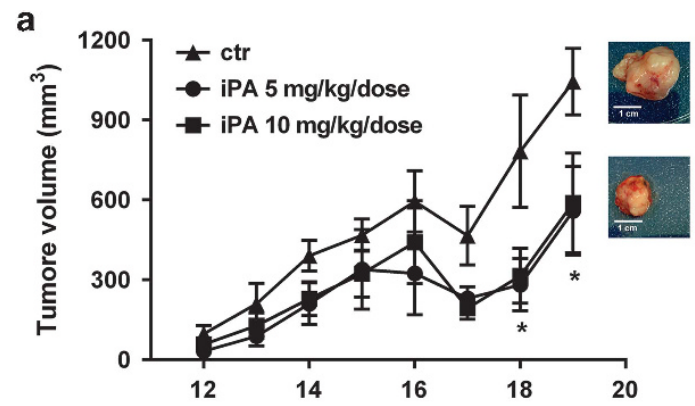

b
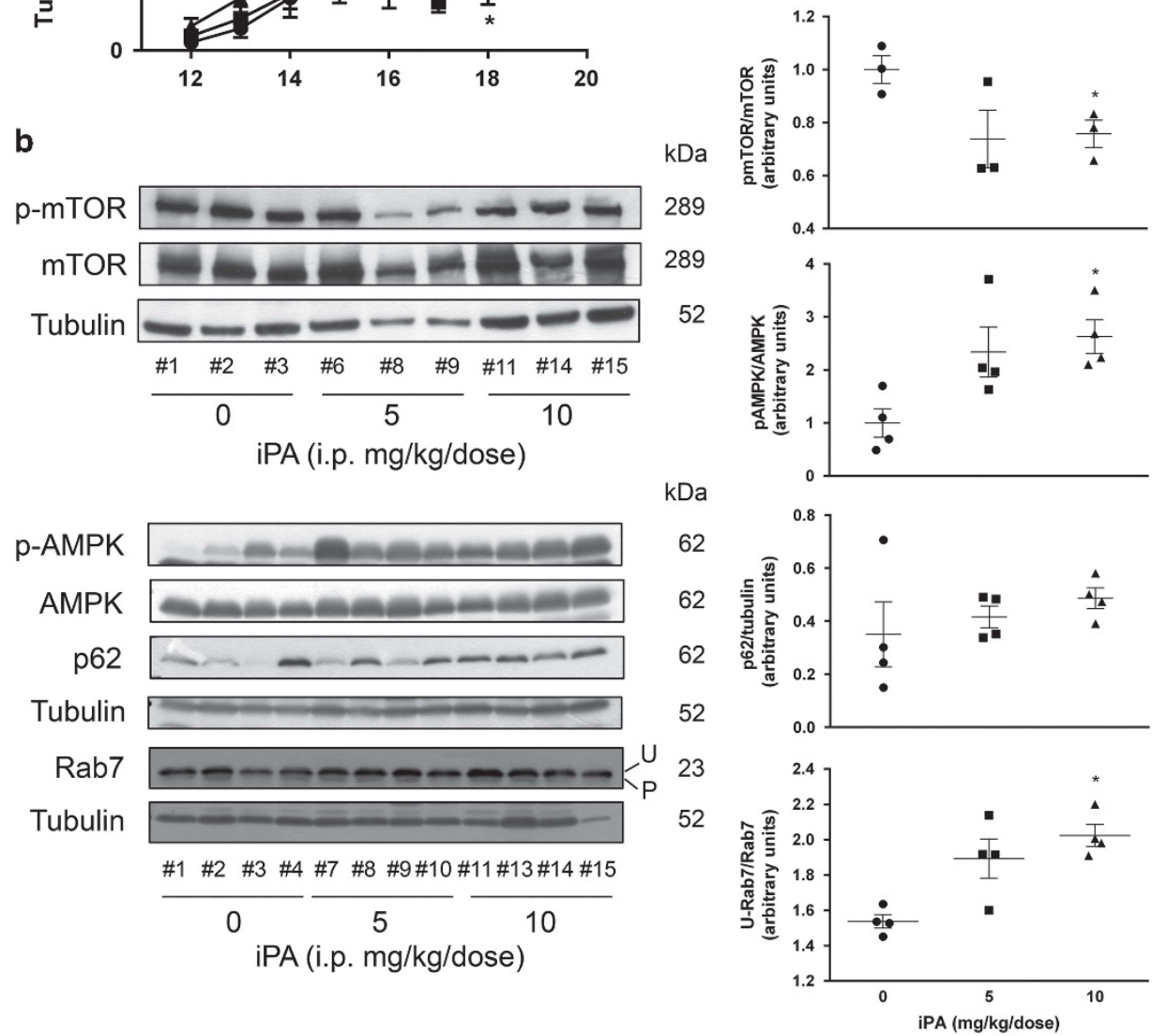

Figure 7 iPA inhibits tumor growth in vivo in melanoma xenografts through the inhibition of the autophagic flux. (a) Female SCID mice were inoculated subcutaneously with A375 cells $\left(2 \times 10^{6}\right.$ cells) and when tumor size reached about $50-70 \mathrm{~mm}^{3}$ the mice were divided into three treatment groups and treated with iPA (5 or $\left.10 \mathrm{mg} / \mathrm{kg} / \mathrm{dose}\right)$ or PBS alone every day by peritumoral injection for 3 weeks. The growth tumor curves were determined by measuring tumor volume expressed as mean tumor size \pm S.E.M. at various time points (ANOVA, $\left.{ }^{*} P<0.05\right)$. Representative pictures of the excised tumors from control and iPA-treated groups (5 mg/kg/dose) are shown near the curves. Scale bars: $1 \mathrm{~cm}$. (b) Representative western blots showing p-mTOR (Ser-2448), mTOR, p-AMPK (Thr-172), AMPK, p62 and Rab7 expression ( $U=$ unprenylated; $P=$ prenylated) in three or four mice belonging to each group of treatment (control, iPA 5 and $10 \mathrm{mg} / \mathrm{kg} / \mathrm{dose}$ ). Tubulin served as a loading control. The quantitative analysis (arbitrary units) for each protein is showed on the right by the column scatter graphs that report individual data point for each mouse (indicated with $\# M$ ( for each group (ANOVA, $\left.{ }^{*} P<0.05\right)$

late stages. ${ }^{43}$ Hence, iPA belongs to a novel class of autophagy modulators able to simultaneously induce the autophagic process upstream and block the autophagic flux downstream, which could be useful as single agents in cancer therapy or in combination with classic chemotherapy with synergistic effects on cell death induction. ${ }^{44}$ The simultaneous activation and inhibition of autophagy has been reported as an interesting approach for chemotherapy sensitization, especially in those tumors with high basal levels of cytoprotective autophagy, as melanoma. ${ }^{45}$ We observed that iPA induces autophagosome accumulation through AMPK activation both in vitro and in vivo. Indeed, as we already reported in endothelial cells, iPA behaves as an AMP mimetic, being phosphorylated by ADK into its 5'monophosphorylated derivative iPAMP, that in turn activates AMPK. ${ }^{25,46}$ AMPK is a key mediator of autophagy, being an energetic sensor that activates autophagy in response to cellular stresses like nutrient deprivation, hypoxia, chemotherapy or targeted therapies, by inhibiting mTOR signaling pathway both in cytoprotective and in cytotoxic autophagy. ${ }^{47}$ Strategies aimed to inhibit in a coordinated manner both mTOR pathway and autophagy showed a promising antitumor effect in melanoma. ${ }^{48}$ Interestingly, we observed that the dual effect of iPA on autophagosome accumulation and autophagic flux inhibition relies on different and independent mechanisms, that are respectively AMPK activation and inhibition of Rab7 prenylation (Figure 8). Indeed, even when AMPK is selectively silenced in melanoma cells through siRNA, Rab7 prenylation 


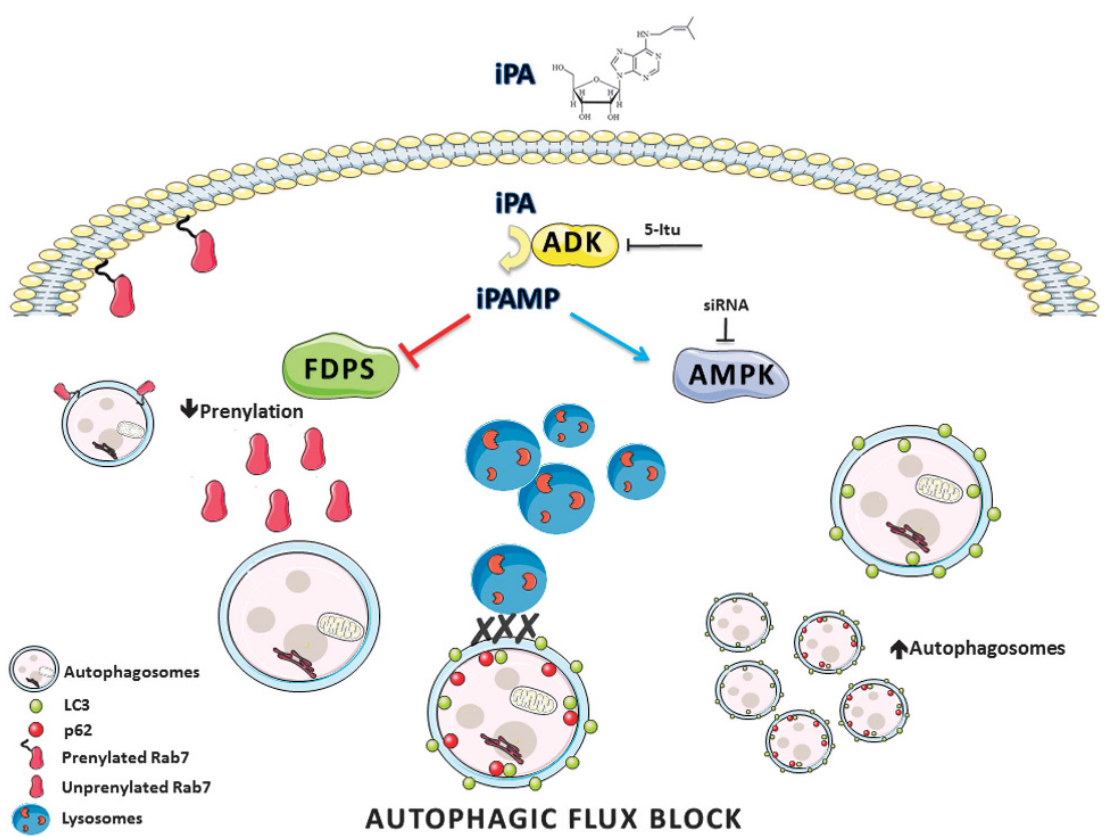

Figure 8 Proposed model of the mechanism by which IPA coordinately activates autophagosome accumulation and inhibits autophagic flux thus leading to melanoma apoptotic cell death

is inhibited upon iPA treatment and hence it remains into the cytoplasm in the inactive form. This is confirmed by the finding that p62 still accumulates when AMPK is silenced, because of the inhibition of basal autophagosome turnover. The apoptotic process, which is activated by iPA following autophagy inhibition, is prevented by AMPK silencing or 5-Itu pretreatment, which acts upstream blocking iPA phosphorylation by ADK, and so is AMPK-dependent. These observations are fundamental to demonstrate the intimate connection among autophagy and apoptosis in the mechanism of action of iPA. Its ability to activate AMPK induces autophagosomes accumulation that are unable to be degraded because of its inhibition of autophagosome clearance, thus becoming detrimental for the cell that induces the apoptotic cell death program, through the intrinsic cascade, confirmed by caspase- 9 activation followed by caspase-3 and PARP cleavage. Moreover, iPA does not impair lysosomal functionality, thus allowing the involvement of lysosome proteases in iPA-induced cell death. Autophagy is induced by chemo- and radiotherapy to protect cells from apoptosis and inhibition of autophagy enhances the antitumor effect inducing the apoptotic process. On the other hand, several antitumor treatments have been reported to induce an autophagic cell death independently from apoptosis, especially in those tumors that are resistant to apoptosis. ${ }^{49}$ The combinatorial strategy with cytotoxic drugs is functional to impede or reverse chemotherapy resistance, overcoming autophagy-mediated cytoprotection in cancer therapy Interestingly, compounds like iPA able to induce autophagy simultaneously being cytotoxic through the impairment of autophagosomes final degradation could be also useful as standalone agents in therapy.

The results of our study strongly support the advantage of therapeutic targeting of autophagy in melanoma and provide the preclinical rational to further investigate the antitumor action of iPA, a novel compound that thanks to its peculiar structure is able to induce autophagosome accumulation inhibiting at the same time the autophagic flux. This property may be particularly useful for the selective killing of those tumors, like melanoma, that frequently develop chemotherapy resistance due to protective autophagy activation.

\section{Materials and Methods}

Cell lines and cell culture. Human malignant melanoma cell line A375 was purchased from American Type Culture Collection (ATCC, Manassas, VA, USA). Cells were maintained in DMEM high glucose (Sigma-Aldrich, St. Louis, MO, USA) supplemented with $10 \%$ heat-inactivated FBS (EuroClone, Milan, Italy) and $2 \mathrm{mM} \mathrm{L-}$ glutamine. Cells were cultured at $37^{\circ} \mathrm{C}$ in a humidified $5 \% \mathrm{CO}_{2}$ atmosphere.

Reagents and antibodies. $\mathrm{PA}$ and hydroxychloroquine (HCQ) were provided by Sigma-Aldrich. 5-lodotubercidin (5-ltu) was from Tocris Bioscience (Bristol, UK). Compound C (CC) was provided by Selleck Chemicals (Munich, Germany). The plasmids eGFP-LC3, RLuc-LC3wt and RLuc-LC3G120A were a kind gift of Marja Jäättelä (Danish Cancer Society Research Center, Copenhagen, Denmark). The mRFP-EGFP-LC3 plasmid was obtained from Addgene (Addgene, Cambridge, MA, USA; 21074). The antibodies used were: anti-phospho-mTOR (Cell Signaling Technology, Leiden, The Netherlands; 5536), anti-mTOR (Cell Signaling Technology; 2983), anti-phospho p70 (Cell Signaling Technology; 9208), antiphospho AMPK (Cell Signaling Technology; 2535), anti-AMPK (Cell Signaling Technology; 2603), anti-LC3B (Cell Signaling Technology; 2775), anti-p62/SQTM1 (Abcam, Cambridge, UK; 109012), anti-cleaved-caspase-3 (Cell Signaling Technology; 9664), anti-caspase-3 (Cell Signaling Technology; 9662), anticleaved-caspase-9 (Cell Signaling Technology; 7237), anti-caspase-9 (Cell Signaling Technology; 9502), anti-cleaved-PARP (Cell Signaling Technology; 5625), anti-PARP (Cell Signaling Technology; 9542), anti-Rab7 (Cell Signaling Technology; 9367), anti-Rap1A (Santa Cruz Biotechnology, Dallas, TX, USA; 1482), anti-Rap1 (Santa Cruz Biotechnology; 65), anti-HDJ-2 (Thermo Fisher Scientific, Waltham, MA, USA; MS-225) and anti-alpha tubulin (Sigma-Aldrich).

Drug treatments. iPA powder was dissolved in DMSO $(20 \mathrm{mM})$ and added to cell cultures at indicated concentration. For each experiment, fresh dilutions were made from the stock solution and added to cell cultures at the indicated concentrations in serum-containing medium. 5-Itu was used in pre-treatment at a 
final concentration of $30 \mathrm{nM}$ for $30 \mathrm{~min}$. HCQ was prepared in DMSO $(10 \mathrm{mM})$ and used at indicated concentration. CC was used in pre-treatment for $1 \mathrm{~h}$ at the indicated concentrations.

Proliferation assay. Melanoma cells proliferation was evaluated by measuring BrdU incorporation into DNA (BrdU colorimetric assay kit; Roche Applied Science, South San Francisco, CA, USA) of replicating cells (during the S-phase of the cell cycle). Cells $\left(8 \times 10^{3} /\right.$ well) were seeded into 96 -well plates and treated for 24,48 or $72 \mathrm{~h}$. Newly synthesized BrdU-DNA was determined using an ELISA reader at $450 \mathrm{~nm}$ (Thermo Fisher Scientific). All experiments were performed in triplicate, and the relative cell viability was expressed as a percentage comparison with the untreated control cells. The $\mathrm{IC}_{50}$ was calculated by nonlinear regression analysis using the GraphPad Prism 6.0 software (GraphPad Software Inc., La Jolla, CA, USA).

Colony formation assay. Melanoma cells were seeded in six-well plates (200 cells/well) and treated at time $0 \mathrm{~h}$ with increasing concentrations of IPA alone $(1-10 \mu \mathrm{M})$ with or without the pre-treatment with 5 -ltu $(30 \mathrm{nM}, 30 \mathrm{~min})$ and monitored for $10 \mathrm{n}$ days. Colonies were fixed and stained with crystal violet $(0.5 \% \mathrm{w} / \mathrm{v}$ in methanol $20 \%)$, photographed and counted.

Cell cycle analysis. Melanoma cells were plated in 100-mm dishes. To synchronize cells at the $\mathrm{G} 1 / \mathrm{S}$ interface, they were serum starved in medium with $0.5 \%$ serum for $18 \mathrm{~h}$. Cells pre-treated or not with 5 -Itu ( $30 \mathrm{nM}, 30 \mathrm{~min}$ ) were treated with iPA. After $24 \mathrm{~h}$, the cells were collected, fixed in $70 \%$ ethanol and kept at $-20^{\circ} \mathrm{C}$ overnight. Propidium iodide (PI; $50 \mu \mathrm{g} / \mathrm{ml}$ ) in PBS containing $100 \mathrm{U} / \mathrm{ml}$ DNase-free RNase was added to the cells for $15 \mathrm{~min}$ at room temperature. The cells were acquired by a FACSCalibur flow cytometer (BD Biosciences, San Jose, CA, USA). The analysis was performed with ModFit LT v3.2 (Verity Software House, Inc., Topsham, ME, USA); 10000 events, corrected for debris and aggregate populations, were collected.

Apoptosis analysis. Briefly, cells grown in six-well culture plates were harvested using trypsin and washed in PBS. Then, the cells were resuspended in Annexin V binding buffer (10 mM HEPES/NaOH, pH 7.4, $140 \mathrm{mM} \mathrm{NaCl}, 2.5 \mathrm{mM}$ $\mathrm{CaCl}_{2}$ ) and stained with Annexin V-FITC (BMS147FI; eBioscience, San Diego, CA, USA) for $20 \mathrm{~min}$ and after with $\mathrm{PI}$ at room temperature for additional $15 \mathrm{~min}$ in the dark. The cells were acquired by flow cytometer (BD Biosciences) within $1 \mathrm{~h}$ after staining. At least 30000 events were collected, and the data were analyzed by CellQuest Pro software (BD Biosciences).

Flow cytometric analysis of autophagy. Melanoma cells were grown in six-well culture plates and incubated with drugs, iPA, 5-Itu and chloroquine as indicated in the text. After, cells were washed with PBS, harvested with trypsin and washed in PBS. LysoTracker Red DND-99 (excitation/emission, 577/590 nm) was added to melanoma cells at a final concentration of $50 \mathrm{nM}$ for $30 \mathrm{~min}$ at $37^{\circ} \mathrm{C}$. The quantification of acidic vesicular organelles (AVO), as a marker of autophagy, was detected by staining melanoma cells with lysosomotropic agent acridine orange $(2 \mu \mathrm{g} / \mathrm{ml})$ for $15 \mathrm{~min}$ at $37^{\circ} \mathrm{C}$. The cells were acquired by a flow cytometer. At least 30000 events were collected and the data were analyzed by FlowJo software (FlowJo LLC, Ashland, OR, USA) or by Cell- Quest Pro software (BD Biosciences).

Western blot. After treatment, A375 cells were washed with PBS, detached using trypsin and collected with ice-cold RIPA Buffer $(50 \mathrm{mM}$ Tris-HCl, $150 \mathrm{mM}$ $\mathrm{NaCl}, 0.5 \%$ Triton $\mathrm{X}-100,0.5 \%$ deoxycholic acid, $10 \mathrm{mg} / \mathrm{ml}$ leupeptin, $2 \mathrm{mM}$ phenylmethylsulfonyl fluoride and $10 \mathrm{mg} / \mathrm{ml}$ aprotinin). Cell debris were removed by centrifugation (14500 g for $20 \mathrm{~min}$ at $4^{\circ} \mathrm{C}$ ) and then the protein concentration was determined by Biorad Protein Assay (Biorad, Richmond, CA, USA). About 15 to $30 \mu \mathrm{g}$ of proteins were resuspended in sample buffer, loaded onto SDSpolyacrylamide gels under reducing conditions and then transferred to nitrocellulose membranes. The membranes were blocked with $5 \%$ non-fat dry milk (Biorad) and incubated with the specific antibodies overnight at $4{ }^{\circ} \mathrm{C}$. Immunodetection of specific proteins was carried out with horseradish peroxidase-conjugated secondary antibody for $1 \mathrm{~h}$ at room temperature. The membranes were stained by using the enhanced chemiluminescence system (Amersham Biosciences, Amersham, UK). If necessary, the membranes were stripped and reprobed with another antibody. Immunoreactive bands were quantified using ImageJ software (US National Institutes of Health, Bethesda, MD, USA). The intensity of the staining of $\alpha$-tubulin (using $\alpha$-tubulin monoclonal antibody; Sigma-Aldrich) and the total amount of each of the studied proteins were used as loading controls for data normalization.

Cathepsin assay. The activity of lysosomal cathepsin proteases was determined using the Cathepsin B Activity Assay kit (BioVision, Milpitas, CA, USA). Briefly, $50 \mu \mathrm{g}$ of cell lysates were resuspended in Cathepsin B Cell Lysis Buffer and then the Cathepsin B Reaction Buffer was added to each sample following the manufacturer's protocol. The activity of Cathepsin B was expressed as fold-increase in relative fluorescence units (RFU) compared to the level of negative control sample.

Transfections. For the reporter assay in living cells, melanoma cells were transient transfected with $5 \mu \mathrm{g} / \mu \mathrm{l}$ of RLuc-LC3 ${ }^{\mathrm{wt}}$ and RLuc-LC3 ${ }^{\mathrm{G} 120 \mathrm{~A}}$ in $1 \times 10^{6}$ cells using Nucleofector (Lonza, Basel, Switzerland) according to the manufacturer's instructions.

Transient transfection of siRNA was performed with RNAiMAX (Invitrogen, Thermo Fisher Scientific, Waltham, MA, USA) according to the manufacturer's instructions using $200 \mathrm{nM}$ of siRNA for AMPK.

RLuc reporter assay for autophagic flux measurement. RLuc reporter assays for autophagic flux were performed in live cells as described. ${ }^{27}$ Melanoma cells were transfected with RLuc-LC3 ${ }^{\text {wt }}$ and RLuc-LC3 ${ }^{\mathrm{G} 120 \mathrm{~A}}$ and then were plated in white 96-well plates. The following day, medium was replaced with fresh culture medium and the cells were treated with different concentrations of IPA. After $24 \mathrm{~h}, 60 \mathrm{nM}$ of Enduren substrate (Promega, Madison, WI, USA; E6481) dissolved in medium was added to the cells. RLucLC3 activity was assessed in kinetic measurement using the EnSpire Multilabel Plate Reader (PerkinElmer, Waltham, MA, USA). The luminescence measurement was performed at indicated intervals of 2, 4 and $12 \mathrm{~h}$. Finally the luminescence was determined as the ratio of RLucLC3 ${ }^{\text {wt }}$ and RLuCLC3 ${ }^{\text {mut }}$. These values were normalized to time point zero for a given treatment. The mRFP-EGFP-LC3 construct created by T Yoshimori was used to analyze the autophagic flux through confocal images. Cells were transfected with ptfLC3-expressing plasmid using Lipofectamine 3000 (Invitrogen) according to the manufacturer's instructions and then treated with indicated concentration of IPA for $48 \mathrm{~h}$. After, cells were submitted to confocal procedure.

Confocal microscopy. Melanoma cells were grown on glass coverslips in 12 well plates. After $24 \mathrm{~h}$ cell medium was replaced and cells were treated for $48 \mathrm{~h}$ with IPA at different concentrations (1-10 $\mu \mathrm{M})$. Then, cells were washed with PBS and fixed with $4 \%$ parafolmaldehyde for $15 \mathrm{~min}$; cells were washed in PBS and blocked with blocking solution $(0,1 \%$ Triton, $1 \%$ BSA, $0.02 \%$ sodium azide, $50 \mathrm{mM}$ ammonium chloride) for $20 \mathrm{~min}$ at room temperature in the dark. Afterwards cells were incubated with anti-p62 (Abcam \#109012), Rab7 (Cell Signaling Technology; 9367) and anti-LAMP-1(Abcam \#24170) at a final concentration of $1 \mu \mathrm{g} / \mathrm{ml}$ at room temperature for $1 \mathrm{~h}$. Immunofluorescence staining was obtained by incubating for 90 min with Alexa Fluor 488 donkey anti-rabbit IgG (A31573) and Alexa Fluor 647 donkey anti-rabbit lgG (A21206) secondary antibody at a final concentration of $4 \mu \mathrm{g} /$ $\mathrm{ml}$, respectively (Invitrogen). To evaluate lysosomal functionality cells were labeled with LysoTracker Red $75 \mathrm{nM}$ for $2 \mathrm{~h}$ at $37^{\circ} \mathrm{C}$ and then fixed. The nuclei were counterstained with DAPI (1:2000) and slides were mounted using VectaMount solution ( $A Q$ Vector Laboratories, Burlingame, $C A, U S A)$. Cells were examined under a Leica confocal microscope (Leica Microsystems, Milan, Italy) and then analyzed through ImageJ software as previously described. ${ }^{50}$

\section{In vivo studies}

Animals: Twenty female SCID mice (SHO, 6-8 weeks old) were obtained from Charles River Laboratories Italia (Lecco, Italy). Animals were maintained under clean room conditions in sterile filter top cages with Alpha-Dri bedding and housed on high-efficiency particulate air-filtered ventilated racks. Animals received sterile rodent chow and water ad libitum. All of the experimental procedures were conducted in accordance with the Institute for Laboratory Animal Research Guide for the Care and Use of Laboratory Animals.

Subcutaneous xenograft models in athymic mice: A375 cells $\left(2 \times 10^{6}\right.$, suspended in $200 \mu \mathrm{l}$ of PBS) were implanted subcutaneously into the right flank region of each mouse and allowed to grow to the size of approximately $50-70 \mathrm{~mm}^{3}$. Mice were randomized among and regrouped such that tumor volumes of all groups of mice were in the same range and began to receive treatment. IPA was administered every day by peritumoral injection for 3 weeks at 5 or $10 \mathrm{mg} / \mathrm{kg} / \mathrm{dose}$ in $150 \mu \mathrm{l}$ of PBS. Animals in the corresponding control group received PBS $(150 \mu \mathrm{l})$ 
peritumoral injected on the same schedule as IPA. Mice were daily monitored for clinical signs and mortality. Body weight recordings were carried out bi- or threeweekly. Progress of tumors was daily determined by external two-dimensional caliper measurements, and tumor volumes were calculated using a standard hemiellipsoid formula: (length $(\mathrm{mm}) \times$ width $\left.(\mathrm{mm})^{2}\right) / 2$. Tumor volumes were analyzed using one-way ANOVA. At the end of study, mice were humanely killed and tumors were resected and frozen immediately or fixed in $10 \%$ formalin for further analysis.

For western blot analysis, frozen tumor pieces were disrupted for protein extraction by gentle homogenization (Potter-Elvehjem Pestle) in cold RIPA buffer. After removal of cell debris by centrifugation ( $14500 \mathrm{~g}$ for $20 \mathrm{~min}$ at $4^{\circ} \mathrm{C}$ ), the protein amounts were estimated.

Statistical analysis. Statistical computations were performed using the GraphPad Prism 6.0 software (La Jolla, CA, USA). Data obtained from multiple experiments were calculated as mean \pm S.D. and analyzed for statistical significance by using the two-tailed Student's $t$-test, for independent groups, or ANOVA followed by Tukey correction for multiple comparisons. $P$-values less than 0.05 were considered statistically significant.

\section{Conflict of Interest}

The authors declare no conflict of interest.

Acknowledgements. This study was supported by Associazione Italiana per la Ricerca sul Cancro (AIRC) and Fondazione Cariplo (AIRC TRIDEO 2015 No. 17216 to SP) and by AIRC (IG No. 13312 and No. 18999 to MB). We thank Dr. Donatella Fiore for technical support in animal care.

1. Garbe C, Eigentler TK, Keilholz U, Hauschild A, Kirkwood JM. Systematic review of medical treatment in melanoma: current status and future prospects. Oncologist 2011; 16 : 5-24.

2. Lawrence MS, Stojanov P, Polak P, Kryukov GV, Cibulskis K, Sivachenko A et al. Mutational heterogeneity in cancer and the search for new cancerassociated genes. Nature 2013; 499 214-218.

3. Hodi FS, Chesney J, Pavlick AC, Robert C, Grossmann KF, McDermott DF et al. Combined nivolumab and ipilimumab versus ipilimumab alone in patients with advanced melanoma: 2 year overall survival outcomes in a multicentre, randomised, controlled, phase 2 trial. Lancet Oncol 2016; 17: 1558-1568

4. Polkowska M, Czepielewska E, Kozłowska-Wojciechowska M. Drug combinations as the new standard for melanoma treatment. Curr Treat Opt Oncol 2016; $17: 61$.

5. Redman JM, Gibney GT, Atkins MB. Advances in immunotherapy for melanoma. BMC Med 2016; 14: 20.

6. Welsh SJ, Rizos H, Scolyer RA, Long GV. Resistance to combination BRAF and MEK inhibition in metastatic melanoma: where to next? Eur J Cancer 2016; 62: 76-85.

7. Green DR, Levine $B$. To be or not to be? How selective autophagy and cell death govern cell fate. Cell 2014; 157: 65-75

8. Choi AM, Ryter SW, Levine B. Autophagy in human health and disease. N Engl J Med 2013; 368: $651-662$

9. White $\mathrm{E}$. The role for autophagy in cancer. J Clin Invest 2015; 125: 42-46

10. Liu H, He Z, Simon HU. Targeting autophagy as a potential therapeutic approach for melanoma therapy. Semin Cancer Biol 2013; 23: 352-360.

11. Ma XH, Piao S, Wang D, McAfee QW, Nathanson KL, Lum JJ et al. Measurements of tumor cell autophagy predict invasiveness, resistance to chemotherapy, and survival in melanoma. Clin Cancer Res 2011; 17: 3478-3489.

12. Bifulco M, Malfitano AM, Proto MC, Santoro A, Caruso MG, Laezza C. Biological and pharmacological roles of N6-isopentenyladenosine: an emerging anticancer drug. Antic ancer Agents Med Chem 2008; 8: 200-204.

13. Yang J, Zhang J, Huang Z, Wang Z, Zhu Q, Liu L. Correlation of cytokinin levels in the endosperms and roots with cell number and cell division activity during endosperm development in rice. Ann Bot 2002; 90: 369-377.

14. Laten HM, Zahareas-Doktor $\mathrm{S}$. Presence and source of free isopentenyladenosine in yeasts. Proc Natl Acad Sci USA 1985; 82: 1113-1115.

15. Gillman EC, Slusher LB, Martin NC, Hopper AK. MOD5 translation initiation sites determine N6-isopentenyladenosine modification of mitochondrial and cytoplasmic tRNA. Mol Cell Biol 1991: 11: 2382-2390

16. Spinola M, Galvan A, Pignatiello C, Conti B, Pastorino U, Nicander B et al. Identification and functional characterization of the candidatetumor suppressor gene TRIT1 in human lung cancer. Oncogene 2005; 24: 5502-5509.

17. Moustafa ME, Carlson BA, El-Saadani MA, Kryukov GV, Sun QA, Harney JW et al. Selective inhibition of selenocysteinetRNA maturation and seleno protein synthesis in transgenic mice expressing isopentenyladenosine-deficient selenocysteinetRNA. Mol Cell Biol 2001; 21: 3840-3852.

18. Laezza C, Notarnicola M, Caruso MG, Messa C, Macchia M, Bertini S et al. N6isopentenyladenosine arrests tumor cell proliferation by inhibiting farnesyl diphosphate synthase and protein prenylation. FASEB J 2006; 20: 412-418.

19. Laezza C, Caruso MG, Gentile T, Notarnicola M, Malfitano AM, Di Matola T et al. N6isopentenyladenosine inhibits cell proliferation and induces apoptosis in a human colon cancer cell line DLD1. Int J Cancer 2009; 124: 1322-1329.

20. Laezza C, Malfitano AM, Di Matola T, Ricchi P, Bifulco M. Involvement of Akt/NF-кB pathway in N6-isopentenyladenosine-induced apoptosis in human breast cancer cells. Mol Carcinog 2010; 49: 892-901.

21. Castiglioni S, Casati S, Ottria R, Ciuffreda P, Maier JA. N6-isopentenyladenosine and its analogue N6-benzyladenosine induce cell cycle arrest and apoptosis in bladder carcinoma T24 cells. Anticancer Agents Med Chem 2013; 13: 672-678.

22. Casati S, Ottria R, Baldoli E, Lopez E, Maier JA, Ciuffreda P. Effects of cytokinins, cytokinin ribosides and their analogs on the viability of normal and neoplastic human cells. Anticancer Res 2011; 31: 3401-3406

23. Spinola M, Colombo F, Falvella FS, Dragani TA. N6-isopentenyladenosine: a potential therapeutic agent for a variety of epithelial cancers. Int J Cancer 2007; 120: 2744-2748.

24. Scrima M, Lauro G, Grimaldi M, Di Marino S, Tosco A, Picardi P et al. Structural evidence of N6-isopentenyladenosine as a new ligand of farnesyl pyrophosphate synthase. J Med Chem 2014; 57: 7798-7803.

25. Pisanti S, Picardi P, Ciaglia E, Margarucci L, Ronca R, Giacomini A et al. Antiangiogenic effects of N6-isopentenyladenosine, an endogenous isoprenoid end product, mediated by AMPK activation. FASEB J 2014; 28: 1132-1144.

26. Høyer-Hansen M, Jäättelä M. AMP-activated protein kinase: a universal regulator of autophagy? Autophagy 2007; 3: 381-383.

27. Farkas $T$, Høyer-Hansen $M$, Jäättelä $M$. Identification of novel autophagy regulators by a luciferase-based assay for the kinetics of autophagic flux. Autophagy 2009; 5: 1018-1025.

28. Kimura S, Noda T, Yoshimori T. Dissection of the autophagosome maturation process by a novel reporter protein, tandem fluorescent-tagged LC3. Autophagy 2007; 3: 452-460.

29. Kawai A, Uchiyama H, Takano S, Nakamura N, Ohkuma S. Autophagosome-lysosome fusion depends on the $\mathrm{pH}$ in acidic compartments in $\mathrm{CHO}$ cells. Autophagy 2007; 3: 154-157.

30. Hyttinen JM, Niittykoski M, Salminen A, Kaarniranta K. Maturation of autophagosomes and endosomes: a key role for Rab7. Biochim Biophys Acta 2013; 1833: 503-510.

31. Ciaglia E, Abate M, Laezza C, Pisanti S, Vitale M, Seneca V et al. Antiglioma effects of N6isopentenyladenosine, an endogenous isoprenoid end product, through the downregulation of epidermal growth factor receptor. Int J Cancer 2017; 140: 959-972.

32. Ciaglia E, Pisanti S, Picardi P, Laezza C, Malfitano AM, D'Alessandro A et al. N6isopentenyladenosine, an endogenous isoprenoid end product, directly affects cytotoxic and regulatory functions of human NK cells through FDPS modulation. J Leukoc Biol 2013; 94: 1207-1219.

33. Ciaglia E, Pisanti S, Picardi P, Laezza C, Sosa S, Tubaro A et al. N6-isopentenyladenosine affects cytotoxic activity and cytokines production by IL-2 activated NK cells and exerts topical anti-inflammatory activity in mice. Pharmacol Res 2014; 89: 1-10.

34. Tang DY, Ellis RA, Lovat PE. Prognostic impact of autophagy biomarkers for cutaneous melanoma. Front Oncol 2016; 6: 236

35. Høyer-Hansen M, Jäättelä M. Autophagy: an emerging target for cancer therapy. Autophagy 2008; 4: 574-580.

36. Corazzari M, Fimia GM, Lovat $P$, Piacentini $M$. Why is autophagy important for melanoma? Molecular mechanisms and therapeutic implications. Semin Cancer Biol 2013; 23: 337-343.

37. Lazova R, Camp RL, Klump V, Siddiqui SF, Amaravadi RK, Pawelek JM. Punctate LC3B expression is a common feature of solid tumors and associated with proliferation, metastasis, and poor outcome. Clin Cancer Res 2012; 18: 370-379.

38. Yang ZJ, Chee CE, Huang S, Sinicrope FA. The role of autophagy in cancer: therapeutic implications. Mol Cancer Ther 2011; 10: 1533-1541.

39. Rangwala R, Leone R, Chang YC, Fecher LA, Schuchter LM, Kramer A et al. Phase I trial of hydroxychloroquine with dose-intense temozolomide in patients with advanced solid tumors and melanoma. Autophagy 2014; 10: 1369-1379.

40. Zhao X, Fang Y, Yang Y, Qin Y, Wu P, Wang T et al. Elaiophylin, a novel autophagy inhibitor, exerts antitumor activity as a single agent in ovarian cancer cells. Autophagy 2015; 11 : 1849-1863.

41. Gutierrez MG, Munafó DB, Berón W, Colombo Ml. Rab7 is required for the normal progression of the autophagic pathway in mammalian cells. J Cell Sci 2004; 117: 2687-2697.

42. Dykstra KM, Allen C, Born EJ, Tong H, Holstein SA. Mechanisms for autophagy modulation by isoprenoid biosynthetic pathway inhibitors in multiple myeloma cells. Oncotarget 2015; 6: 41535-41549.

43. Miettinen TP, Björklund M. Mevalonate pathway regulates cell size homeostasis and proteostasis through autophagy. Cell Rep 2015; 13: 2610-2620.

44. Li M, Yang Z, Vollmer LL, Gao Y, Fu Y, Liu C et al. AMDE-1 is a dual function chemical for autophagy activation and inhibition. PLOS ONE 2015; 10: e0122083.

45. Chi KH, Wang YS, Huang YC, Chiang HC, Chi MS, Chi CH et al. Simultaneous activation and inhibition of autophagy sensitizes cancer cells to chemotherapy. Oncotarget 2016; 7 : 58075-58088. 
46. Hardie DG. AMP-activated/SNF1 protein kinases: conserved guardians of cellular energy. Nat Rev Mol Cell Biol 2007; 8: 774-785.

47. Kim J, Kundu M, Viollet B, Guan KL. AMPK and mTOR regulate autophagy through direct phosphorylation of Ulk1. Nat Cell Biol 2011; 13: 132-141.

48. Xie X, White EP, Mehnert JM. Coordinate autophagy and mTOR pathway inhibition enhances cell death in melanoma. PLOS ONE 2013; 8: e55096.
49. Maiuri MC, Zalckvar E, Kimchi A, Kroemer G. Self-eating and self-killing: crosstalk between autophagy and apoptosis. Nat Rev Mol Cell Biol 2007; 8: 741-752.

50. Amodio G, Venditti R, De Matteis MA, Moltedo O, Pignataro P, Remondelli P. Endoplasmic reticulum stress reduces COPII vesicle formation and modifies Sec23a cycling at ERESs. FEBS Lett 2013; 587: 3261-3266.

Supplementary Information accompanies this paper on Cell Death and Differentiation website (http://www.nature.com/cdd) 